\title{
Genetic Diversity and DNA Barcoding of Yam Accessions from Southern Nigeria
}

\author{
George N. Ude ${ }^{1}$, David O. Igwe ${ }^{1,2}$, Julian McCormick ${ }^{1}$, Onyinye Ozokonkwo-Alor ${ }^{3}$, \\ Jonathan Harper'1, Daniel Ballah', Cecille Aninweze ${ }^{3}$, Obih Chosen', Michael Okoro ${ }^{3,4}$, \\ Christabel Ene ${ }^{3}$, Venatus Chieze ${ }^{3}$, Mariam Unachukwu ${ }^{3}$, Christie Onyia ${ }^{3}$, George Acquaah', \\ James Ogbonna ${ }^{3,5}$, Aditi Das ${ }^{1}$
}

\author{
${ }^{1}$ Department of Natural Sciences, Bowie State University, Bowie, USA \\ ${ }^{2}$ Department of Biotechnology, Faculty of Science, Ebonyi State University, Abakaliki, Nigeria \\ ${ }^{3}$ Godfrey Okoye University, Enugu, Nigeria \\ ${ }^{4}$ DNA Learning Center, Cold Spring Harbor, NYC, USA \\ ${ }^{5}$ Department of Microbiology, University of Nigeria, Nsukka, Nigeria \\ Email: ^gude@bowiestate.edu
}

How to cite this paper: Ude, G.N., Igwe, D.O., McCormick, J., Ozokonkwo-Alor, O., Harper, J., Ballah, D., Aninweze, C., Chosen, O., Okoro, M., Ene, C., Chieze, V., Unachukwu, M., Onyia, C., Acquaah, G., Ogbonna, J. and Das, A. (2019) Genetic Diversity and DNA Barcoding of Yam Accessions from Southern Nigeria. American Journal of Plant Sciences, 10, 179-207. https://doi.org/10.4236/ajps.2019.101015

Received: December 21, 2018

Accepted: January 19, 2019

Published: January 22, 2019

Copyright () 2019 by author(s) and Scientific Research Publishing Inc. This work is licensed under the Creative Commons Attribution International License (CC BY 4.0).

http://creativecommons.org/licenses/by/4.0/

(c) (7) Open Access

\begin{abstract}
Knowledge of genetic diversity and barcoding of yam is lacking in Enugu and Ebonyi States of southern Nigeria. Therefore, DNA barcoding was used to facilitate identification and biodiversity studies of yam species from Southern Nigeria. Seventy five yam accessions were collected from Enugu and Ebonyi States, including International Institute of Tropical Agriculture for DNA extraction and amplification using a chloroplast DNA (cpDNA) ribulose-1,5bisphosphate carboxylase $(r b c \mathrm{~L})$ marker. There was high level of similarity among the accessions and presence of 534 conserved and 7 variable sites. A transversional mutation of $\mathrm{G} / \mathrm{T}$ at a consensus position of 335 was identified followed by transitions at $362(\mathrm{~A} / \mathrm{G}), 368(\mathrm{~A} / \mathrm{G}), 371(\mathrm{C} / \mathrm{T})$ and $391(\mathrm{C} / \mathrm{T})$ within the accessions. Phylogeny resolved the yam accessions into ten major groups with their bootstrap values ranging from 0 - 100. Phylogenetic diversity was highest in group X, followed by VII, VI and IX. The inter-group genetic distance based on Kimura 2-parameter model ranged from $0.5000 \pm$ $0.4770-5.0560 \pm 2.5760$, while the intra-group had $0.5250 \pm 0.5000-2.0103$ \pm 1.2579 . The mean genetic diversity within the entire population was 0.7970 \pm 0.06910 . BLAST analysis of total bit score, query coverage, and percentage identity were in the ranges of $411-1011,99 \%-100 \%$ and $97 \%-100 \%$, respectively. However, the $r b c \mathrm{~L}$ could not resolve the yam accessions well following the comparative assessment of some discrepancies in the detected number of species from phylogenetic groupings, genetic diversity indices and NCBI BLAST hits, thereby, exposing the inefficiency of this marker in dis-
\end{abstract}


criminating the yam accessions. It was demonstrated that $r b c \mathrm{~L}$ is not an effective marker; therefore, it should not be recommended as a standard-alone marker of choice for DNA barcoding of yam accessions, especially, when accurate identification, discrimination and estimation of genetic diversity of this vital crop are of paramount importance for crop improvement and germplasm conservation.

\section{Keywords}

BLAST, Kimura 2-Parameter, Phylogenetic Diversity, rbcL, Transitional Mutation

\section{Introduction}

Yam (Dioscorea spp.) is a monocotyledonous, an annual or perennial stem tuber belonging to the family Dioscoreaceae of flowering plants. Dioscorea has been described as the largest genus with an estimated 600 species, 10 of which are cultivated and of economic importance [1] [2] [3]. It is the second most important crop after cassava in West Africa [4] [5] [6]. Important and cultivatable species of this vital crop include $D$. cayenensis Lam., D. alata L., D. rotundata Poir., $D$. trifida L. f., D. bulbifera L., D. pentaphylla L., D. opposita Thunb., D. transversa R. Br., D. nummularia Lam. and D. esculenta (Lour.) Burkill. [7]. Within Africa, the common species cultivated include $D$. rotundata (white yam), D. alata (water yam) and D. cayenensis (yellow yam), some of which have been reported to possess medicinal and ornamental values [8].

The crop ranks fourth after potato, sweet potato and cassava as the most important food tuber crop in the world [6]. Yam is important in the economic and social life of people in West Africa [9] [10]. As a starchy food, it provides a major source of cheap caloric energy food for millions of people in the tropical and sub-tropical regions of the world particularly in West Africa, the Caribbean, parts of Asia, South and Central America and the Pacific [8] [11]. Yam tubers are rich sources of energy, vitamin $\mathrm{C}$, musin (glycoprotein), minerals (K, P, Ca, $\mathrm{Mg}, \mathrm{Fe}, \mathrm{Cu}, \mathrm{Co}$ ), phytosterols and steroidal saponins [12]. They are converted into different types of food products such as pounded yam, boiled yam, roasted yam, fried yam slices, yam balls, mashed yams, yam chips, and yam flakes [13]. Fresh yam tubers are also peeled, chipped, dried, and milled into flour that is used to prepare dough called "amala" or "telibowo" [14].

Yams are widespread in the tropics and subtropics. Nigeria is the leading producer of yam with $71 \%$ of the world production [15] [16] [17]. West Africa accounts for over $92 \%$ of the world's production (54.2 million tonnes) [6]. In Ghana and Nigeria, $26.2 \%$ and $31.8 \%$ of people, respectively rely on yam species for income generation and food security [4]. Despite the increasing demand for local consumption and export of yams, there has been a marginal decline in its production due to lack of proper identification of unique species for biodiversity 
diversification for resistance to drastic changes in climate, introgression, cross hybridization and conservation processes to reduce genetic erosion [18]-[24]. To have adequate knowledge of these yam accessions, characterization to the species level, genetic richness and assessment of phylogenetic diversity (PD) are of utmost importance following the genetic resource preservation roles of PD in crop extinction [25], functionality in ecosystems [26] [27] and abiotic variability [28] and these can be achievable with accurate, sensitive and reliable methods.

Morphotaxonomy, the use of morphological characters to identify and classify plants, is currently the most widely used in yams in Nigeria. It entails using traits such as size, form and number of tubers per plant, bulbil formation, presence of spines on the stem, twining direction, fruit shape, and aerial bulbils, which could lead to misidentification of yam species [1] [3] [29]. Further, morphotaxonomy-based method requires cumbersome assessment of whole plants and the importance of this approach declines when specimens/tissue materials are utilized [30]. Use of molecular markers has become significant for accurate identification of these yams to the species level and to harness the genetic diversity inherent in them. Different markers including Restriction fragment length polymorphism (RFLP) [31], Random amplified polymorphic DNA (RAPD) [32], Simple sequence repeat (SSR) [32], Inter-simple repeat (ISSR) [12] and Amplified fragment length polymorphism (AFLP) [32] and gene sequencing [33] [34] have been applied in the characterization of yam species. The use of molecular tools to support morphotaxonomy-based identification is important to clear ambiguous species classification.

A DNA barcode facilitates taxonomic identification through the use of a standardized short genomic segment that is generally found in target lineages with adequate variations capable of discriminating living animals to the species level [35]. DNA barcoding techniques are useful tools in characterization as they allow more objective and rapid specimen identification, which can be cost-effective in providing a central catalog of species diversity. In general, DNA barcoding can improve biodiversity and genetic resource databases [36] [37]. Also, a phylogenetic diversity (PD) method possesses the merits of ease of reconstruction of phylogenetic relationships of species and as such it has resultant potential to enlighten effective taxonomic challenges [38]. MatK and rbcL which are the two plant barcode loci have been chosen for phylogenetic studies of Dioscorea [1] [39]. In this study, a barcoding marker of rbcL was used for identification and genetic characterization of Dioscorea accessions cultivated in southern Nigeria.

\section{Materials and Methods}

\subsection{Sample Collection}

Different yams were sampled from different locations across Eastern and Western Nigerian, including the ones in the germplasm collection at the International Institute of Tropical Agriculture (IITA), Ibadan, Nigeria (Table 1). A total of 
Table 1. List of yam samples collected from different locations and used for DNA barcoding.

\begin{tabular}{|c|c|c|c|}
\hline Sample IDs & Location & LGA & State \\
\hline 1_TDa85.00250 & IITA & Akinyele & Oyo \\
\hline 3_TDa3050 & IITA & Akinyele & Oyo \\
\hline 4_TDb3050 & IITA & Akinyele & Oyo \\
\hline 5_TDb3044 & IITA & Akinyele & Oyo \\
\hline 6_TDb2857 & IITA & Akinyele & Oyo \\
\hline 7_TDb3058 & IITA & Akinyele & Oyo \\
\hline 8_TDb3690 & IITA & Akinyele & Oyo \\
\hline 9_TDd3101 & IITA & Akinyele & Oyo \\
\hline 10_TDd3829 & IITA & Akinyele & Oyo \\
\hline 11_TDd3935 & IITA & Akinyele & Oyo \\
\hline 12_TDd08-38-53 & IITA & Akinyele & Oyо \\
\hline 13_TDdYellow & IITA & Akinyele & Oyo \\
\hline 14_TDd3100 & IITA & Akinyele & Oyо \\
\hline 15_TDc0471-2 & IITA & Akinyele & Oyo \\
\hline 16_TDc0497-4 & IITA & Akinyele & Oyо \\
\hline 17_TDc2813 & IITA & Akinyele & Oyо \\
\hline 18_TDc2796 & IITA & Akinyele & Oyo \\
\hline 19_TDc2792 & IITA & Akinyele & Oyo \\
\hline 20_TDc03-5 & IITA & Akinyele & Oyo \\
\hline 21_TDc04-71-2 & IITA & Akinyele & Oyo \\
\hline 22_TDm2938 & IITA & Akinyele & Oyo \\
\hline 23_TDm3053 & IITA & Akinyele & Oуо \\
\hline 24_TDm3052 & IITA & Akinyele & Oyo \\
\hline 25_TDm3055 & IITA & Akinyele & Oyо \\
\hline 27_TDes3035 & IITA & Akinyele & Oуо \\
\hline 28_TDes3033 & IITA & Akinyele & Oyo \\
\hline 29_TDes 3027 & IITA & Akinyele & Oyo \\
\hline 30_TDes 3030 & IITA & Akinyele & Oyo \\
\hline 31_TDesculenta & IITA & Akinyele & Oyo \\
\hline 33_TDaNwokporo & IITA & Akinyele & Oyo \\
\hline 34_Adakavariety & IITA & Akinyele & Oyo \\
\hline 35_Pepa & IITA & Akinyele & Oyo \\
\hline 36_Ke-emi & IITA & Akinyele & Oyo \\
\hline 37_Ame & IITA & Akinyele & Oyo \\
\hline 38_TDr 89.002665 & IITA & Akinyele & Oyo \\
\hline 39_AlataTda 98.01176 & IITA & Akinyele & Oyo \\
\hline 40_TDa00.00.94 41_Alata & IITA & Akinyele & Oyo \\
\hline 41_Tda00.00600 & IITA & Akinyele & Oyo \\
\hline
\end{tabular}




\section{Continued}

\begin{tabular}{|c|c|c|c|}
\hline 42_OgojaVariety.1 & IITA & Akinyele & Oyo \\
\hline 43_Gbangu_Variety.1 & IITA & Akinyele & Oyo \\
\hline 44_ObioturuguVariety.1 & IITA & Akinyele & Oyo \\
\hline 45_AmolaVariety .1 & IITA & Akinyele & Oyo \\
\hline 46_OginiVariety & IITA & Akinyele & Oyo \\
\hline 47_Damieha & IITA & Akinyele & Oyo \\
\hline 48_Aloshivariety.1 & IITA & Akinyele & Oyo \\
\hline 49_IghuUna & Osonu & Ezeagu & Enugu \\
\hline 51_Alata2 & Osonu & Ezeagu & Enugu \\
\hline 52_Ighu_Dumenturum & Osonu & Ezeagu & Enugu \\
\hline 53_Ighu & Osonu & Ezeagu & Enugu \\
\hline 54_IghuUna.2 & Osonu & Ezeagu & Enugu \\
\hline 57_2-WhiteYam-Iyo & Ukaka Ngwo & Enugu North & Enugu \\
\hline 59_D10WhiteNwopoko-Adaka & Agbalenyi Nachi & Oji-River & Enugu \\
\hline 60_D1Water-Nbana1 & Agbalenyi Nachi & Oji-River & Enugu \\
\hline 61-6-EDO & Ukaka Ngwo & Enugu North & Enugu \\
\hline 62_3LeavedYam-Ono & Ukaka Ngwo & Enugu North & Enugu \\
\hline 65_WaterYam.Nbana & Ukaka Ngwo & Enugu North & Enugu \\
\hline 68_9ENEGBE & Ndibinagu Umuaga & Udi & Enugu \\
\hline 71_D1WaterYam-Nbana2 & Agbalenyi Nachi & Oji-River & Enugu \\
\hline 72_1-Water_Yam-_Nbana & Ndibinagu Umuaga & Udi & Enugu \\
\hline 73_Water yamji_mbala & Nkalagu & Ishielu & Ebonyi \\
\hline 76_OnaTDd & Ezzamgbo & Ohaukwu & Ebonyi \\
\hline 78_Obella & Ezzamgbo & Ohaukwu & Ebonyi \\
\hline 80_UtekpeVariety_2 & Ezzamgbo & Ohaukwu & Ebonyi \\
\hline 81_WhiteYam-Nw-opoko & Amaeke Amaigbo Ozalla & Nkanu West & Enugu \\
\hline 82_Yellowyam_Akpukpu & Amaeke Amaigbo Ozalla & Nkanu West & Enugu \\
\hline 83_WaterYam-Mbuna & Amaeke Amaigbo Ozalla & Nkanu West & Enugu \\
\hline 84_BitterYam-Iwu_obe & Amaeke Amaigbo Ozalla & Nkanu West & Enugu \\
\hline 85_AerialYam_Edugbe & Amaeke Amaigbo Ozalla & Nkanu West & Enugu \\
\hline 86_3LeavedYam_Ona & Ede Oballa & Nsukka & Enugu \\
\hline 87_WaterYam-Mbana & $\mathrm{Nru}$ & Nsukka & Enugu \\
\hline 89_WhiteYam_Nwopoko & Ibagwa Aka & Igboeze South & Enugu \\
\hline 90_Yellowyam_Oku & Ihe Owerre & Nsukka & Enugu \\
\hline 91_TrifoliateYam_TDb & Ukana & Udi & Enugu \\
\hline 92_ChineseYam_TDes & Ukana & Udi & Enugu \\
\hline 93_YellowYam_TDes & Ukana & Udi & Enugu \\
\hline
\end{tabular}

IITA = International Institute of Tropical Agriculture; LGA = Local Government Area. 
eleven Local Government Areas (LGAs), cutting across three States including Oyo (where IITA, Ibadan is located), Enugu and Ebonyi States were used for the yam collection (Figure 1). The IITA, Ibadan, has Genetic Resources Unit that contains many yam species from other parts of Nigeria.

\subsection{DNA Extraction}

Fresh young leaves of yam species weighing from $0.1-0.2 \mathrm{~g}$ were collected for DNA extraction using Silica resin method standardized by the DNA Learning Center (http://www.dnabarcoding101.org/lab/protocol-2.html) [40] In brief, fresh young yam leaf samples were weighed and homogenized in $300 \mu \mathrm{L}$ of lysis solution using sterile mortar and pestle followed by incubation in a heat block at $65^{\circ} \mathrm{C}$ for 10 minutes. Next, samples were centrifuged in a balanced configuration at maximum speed (13,000 rev/min) for $1 \mathrm{~min}$ to pellet debris. A $150 \mu \mathrm{L}$ sample of the supernatant was transferred to fresh micro centrifuge tubes, being careful not to disturb the debris pellet. A $3 \mu \mathrm{L}$ silica resin, was subsequently added to the respective supernatants, mixed well by pipetting up and down, and placed for 5 minutes in a heat block at $57^{\circ} \mathrm{C}$. The silica resin is a DNA binding matrix which in the presence of lysis solution binds readily to nucleic acids. After incubation, tubes were subject to centrifugation, with cap hinges pointing outward, for 30 seconds at maximum speed to pellet the silica resin, which was now bound to nucleic acid. Using a micropipette with fresh tip the supernatant was removed

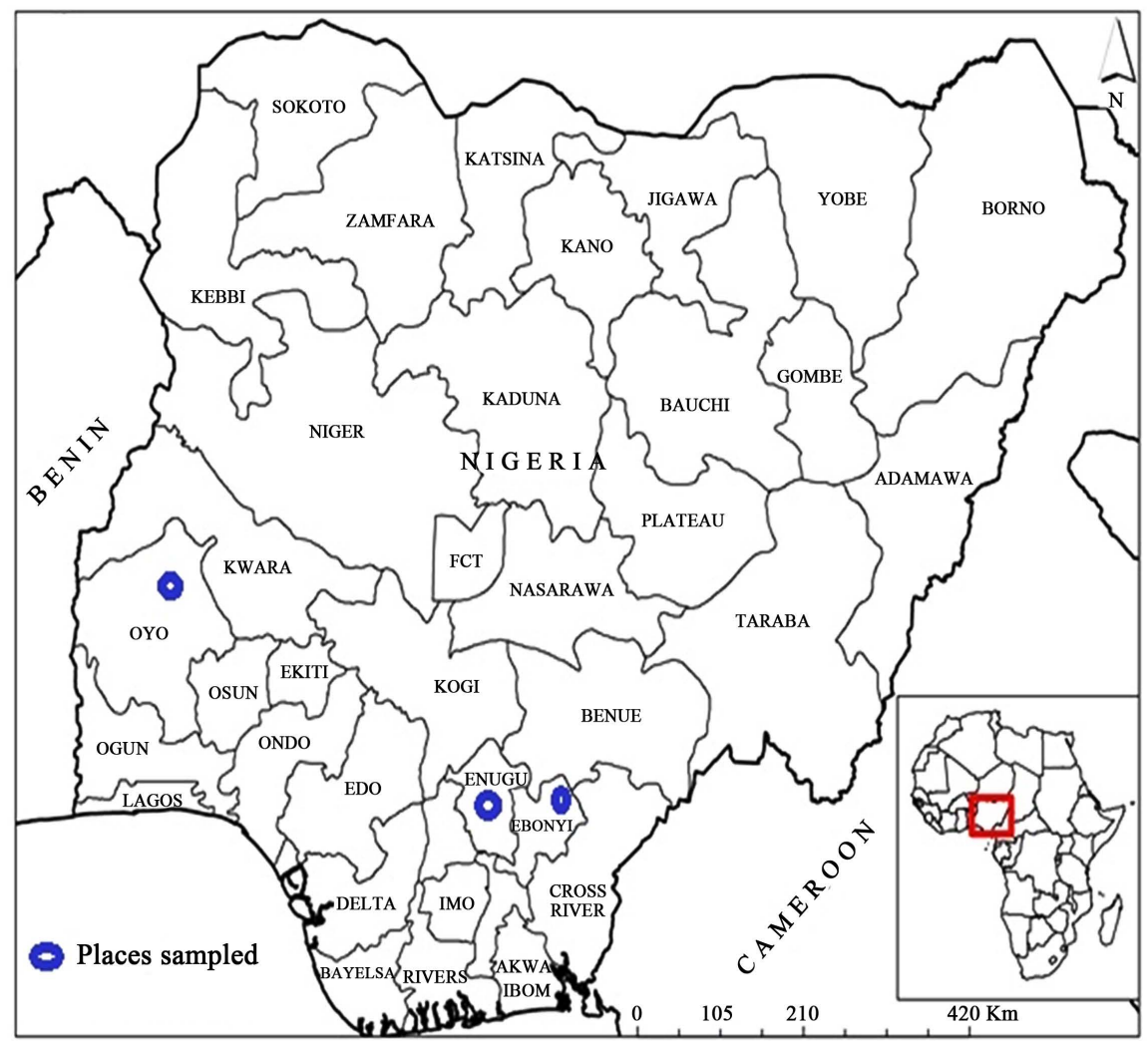

Figure 1. Map of Nigeria showing geographical areas for collection of yam accessions. 
and $500 \mu \mathrm{L}$ of ice cold wash buffer added to the pellet. The silica resin bound to nucleic acid was re-suspended by vortexing and centrifuged to repeat the wash procedure. The wash buffer removes contaminants from the samples while nucleic acids remain bound to the resin. A dry spin step after wash was performed to remove any remnant drops of supernatant with a micropipette. Finally, 100 $\mu \mathrm{L}$ of distilled water was added to the silica resin, mixed well by vortexing and incubated at $57^{\circ} \mathrm{C}$ for 5 minutes. Samples were then centrifuged for 30 seconds at maximum speed to pellet the resin. This time $90 \mu \mathrm{L}$ of the supernatant was transferred to fresh tubes as the nucleic acids eluate from the resin. The eluted DNA was stored to proceed to PCR step.

\subsection{Polymerase Chain Reaction, Agarose Gel Electrophoresis and DNA Sequencing}

PCR amplification was performed using Ready-To-Go PCR beads in a total volume of $25 \mu \mathrm{L}: 2 \mu \mathrm{L}$ of $\sim 100 \mathrm{ng}$ DNA and $23 \mu \mathrm{L}$ of primer/loading dye mix for plant cocktail with rbcL primers (rbcLaf:

5'-TGTAAAACGACGGCCAGTATGTCACCACAAACAGAGACTAAAGC-3' and rbcLa-revM13:

5'-CAGGAAACAGCTATGACGTAAAATCAAGTCCACCRCG-3'). The PCR tubes were placed in a thermal cycler that had been programmed with the appropriate PCR protocol with initial step at $94^{\circ} \mathrm{C}$ for $1 \mathrm{~min}$., 35 cycles of $94^{\circ} \mathrm{C}$ for $15 \mathrm{sec}, 54^{\circ} \mathrm{C}$ for $15 \mathrm{sec}$, and $72^{\circ} \mathrm{C}$ for $30 \mathrm{sec}$., and $8 \mathrm{~min}$ final extension at $72^{\circ} \mathrm{C}$ was maintained. The PCR products or amplicons were electrophoresed in a $1.5 \%$ agarose gel containing $0.5 \mathrm{mg} / \mathrm{ml}$ ethidium bromide and photographed on Transilluminator UV light (Omega G). The generated PCR amplicons sent to Genewiz LLC, New Jersey, USA, for DNA sequencing.

\subsection{Data Analysis}

The sequencing results generated from the Applied Biosystems Genetic automated sequencer (ABI Prism 3130X1, Froster City, CA 94404, USA) at Genewiz LLC were uploaded in the blue line of DNA Subway

(https://dnasubway.cyverse.org/), which is an intuitive interface for analysing DNA barcodes. Using the Blue Line, the assembled sequences were end-trimmed, paired in their respective forward and reverse sequences to build consensus sequences. The consensus sequences from DNA subway were further edited, filtered and assembled for polymorphism detection using BioEdit software (BioEdit sequence aligner editor, version 7.6.2.1). Sequence alignment and percentage similarity searches were compared with GeneBank databases using NCBI web-based site, BLAST. Multiple alignments were done using the ClustalW [41] [42]. Phylogenetic tree reconstruction was performed using MEGA 6 software [43]. Phylogenies were constructed using the Maximum Parsimony and Maximum Likelihood options [44] [45] and the effectiveness of the trees was determined by bootstrapping up to 1000 replicates [46]. 


\section{Results}

\subsection{Sequence Alignment of Sequences Generated from Dioscorea Spp. Using rbcL Barcoding Marker}

A total length of sequence alignment, conserved sites, and variable sites of 525 , 534 and 7 were respectively identified among the sequenced yam species. Different regions of polymorphisms and conserved regions at nucleotide level across the sequences exhibited variations among them. At a position of 335, 62_3LeavedYam_Ono and 76_Ona_TDd possessed a transversional mutation by having $\mathrm{G}$ nucleotide, while other samples had a $\mathrm{T}$ nucleotide (Additional file 1: Figure S1). At a consensus position of 362, yam species such as 3_TDa3050, 4_TDb3050, 5_TDb3044, 6_TDb2857, 7_TDb3058, 8_TDb3690, 61_6-EDO, 83_WaterYam-_Mbuna and 85_AerialYam_Edugbe showed a transitional mutation of A nucleotide, while the rest of the accessions had a $\mathrm{G}$ nucleotide. At a position of 368, accessions such as 1_TDa85.00250, 3_TDa3050, 4_TDb3050, 5_TDb3044, 6_TDb2857, 7_TDb3058, 8_TDb3690, 61_B-6-EDO,

83_WaterYam-_Mbuna, 85_AerialYam_Edugbe had a transitional mutation of A in place of $\mathrm{G}$ nucleotide possessed by other accessions at the same consensus position.. Also at 371 position, accessions including 35_Pepa, 36_Ke-emi, 37_Ame, 38_TDr.89.002665, 39_AlataTda98.01176, 40_TDa00.00.94,

42_OgojaVariety.1, 43_Gbangu_Variety.1, 44_ObioturuguVariety,

45_AmolaVariety.1, 46_OginiVariety, 47_Damieha, 48_Aloshivariety.1, 57_2-WhiteYam_Iyo, 61_6-EDO, 68_9ENEGBE, 78_Obella, 80_UtekpeVariety_2, 81_WhiteYam-Nwoopoko, 82_Yellowyam_Akpukpu, 83_WaterYam-Mbuna, 85_AerialYam_Edugbe, 89_WhiteYam_Nwoopoko and 93_YellowYam_TDes exhibited a transitional mutation by possessing a $\mathrm{C}$ nucleotide, while the remaining species had a T nucleotide. Also at a position of 391, 76_Ona_TDd possessed $\mathrm{C}$, while other remaining yam species had $\mathrm{T}$ nucleotide.

\subsection{Phylogenetic Tree Reconstruction (PTR) and Phylogenetic Diversity (PD)}

Out of the 75 nucleotide sequences used for the analyses, a total of 270 codon positions including $1^{\text {st }}, 2^{\text {nd }}, 3^{\text {rd }}$, and non-coding regions as well as $4.3582 \%$ invariable (monomorphic) sites were found in the final dataset. From the phylogenetic tree analysis, the yam accessions were resolved into ten groups with variable phylogenetic diversities (PDs) (Figure 2). Group I with PD in the range of 0-27 consisted of twenty five accessions including 43_Gbangu_variety, 82_Yellowyam-Akpukpu, 81_Whiteyam-Nwopoko, 89_WhiteYam-Nwopoko, 24_TDm3052, 23_TDm3053, 20_TDc03-5，19_TDc2792, 80_Utekpevariety, 17_TDc2813, 21_TDc04-71-2, 93_Yellowyam-TDes, 18_TDc2796, 68_9ENEGBE, 25_TDm3055, 15_TDc0471-2, 46_Oginivariety, 57_2-Whiteyam-Iyo, 45_Amolavariety, 40_TDa00.00.94, 38_TDr89.002665, 16_TDc0497-4, 78_Obella, 37_Ame and 35_Pepa grouping with D. rotundata obtained from NCBI data with a reference sequence accession of KR072483. The yam accessions were 


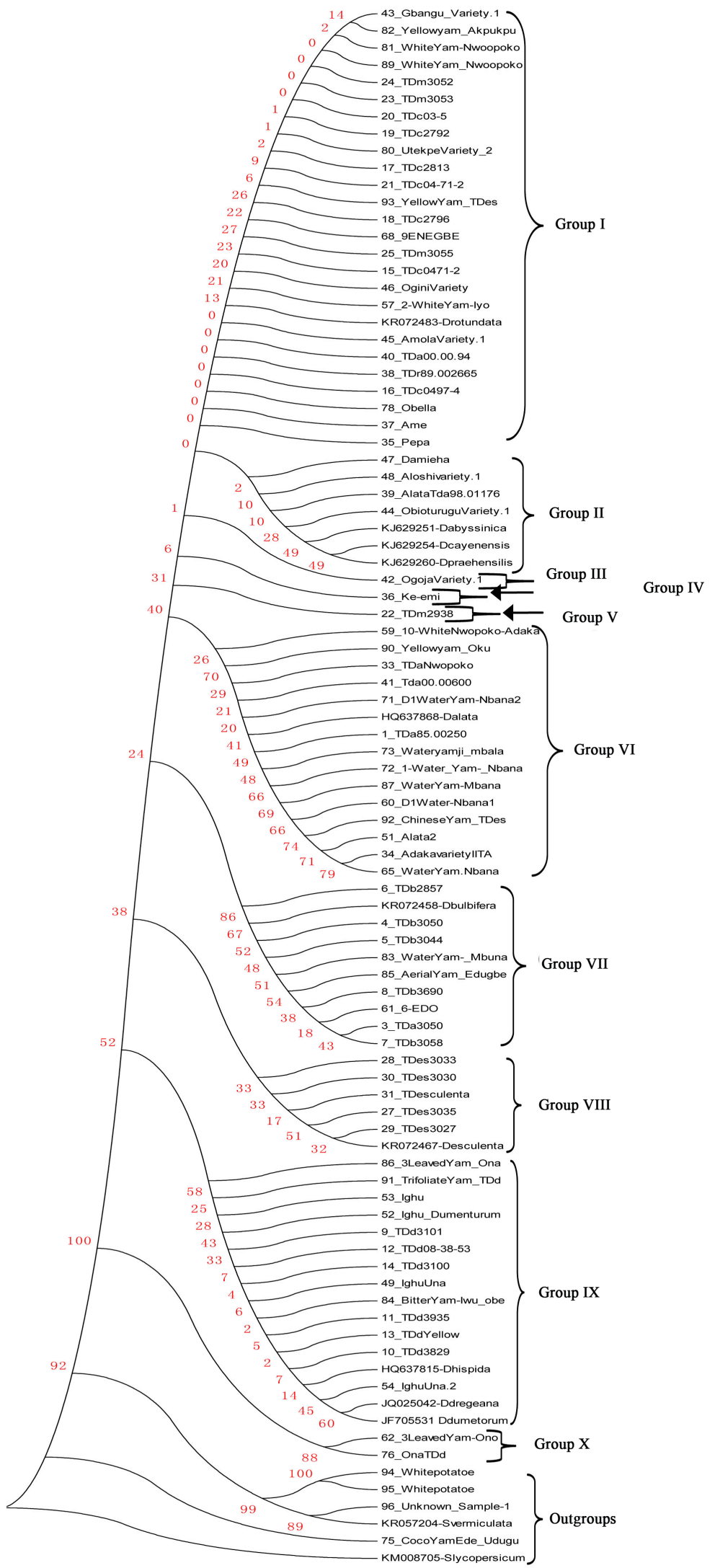

Figure 2. Phylogenetic tree of different yam species as revealed by rbCL barcoding marker. 
collected from different locations including Enugu, Ebonyi and International Institute of Tropical Agriculture (IITA), Ibadan, Nigeria. Group II (with PD of 2-49) contained four accessions such as 47_Damieha, 48_Aloshivariety, 39_Alata TDa98-01176, 44_Obioturuguvariety grouped together KJ629251-D. abyssinica, KJ629254-D. cayenensis and KJ629260-D. praehensillis. Group III (with PD of 1) contained only 42 OOgojavariety.

Groups IV (PD = 6) and V (PD = 31) had 36_Ke-emi and 22_TDm2938, respectively. Group VI (PD = 20 - 79) consisted fourteen including 59_10-Whiteyam-Nwopoko-Adaka, 90_YellowYam-Oku, 33_TDaNwopoko, 41_TDa00.00600, 71_D1WaterYam-Nbana2, 1_TDa85.00250, 73_WaterYam-Mbala, 72_1WaterYam-_Nbana, 87_WaterYam-Mbana, 60_D1WaterYam-Nbana 1, 92_ChineseYam-TDes, 51_Alata2, 34_AdakavarietyIITA, and 65_WaterYam-Nbana that grouped together with $D$. alata retrieved from NCBI database with an accession number of HQ637868. Group VII with PD value in the range of 18-86, had nine accessions including 6_TDb2857, 4_TDb3050, 5_TDb3044, 83_WaterYam-_Mbana, 85_AerialYam- Edugbe, 8_TDb3690, 61_6-Edo, 3_TDa3050 and TDb3058 grouped together with a known $D$. bulbifera species (with an accession No: KR072458) that was retrieved from NCBI database. Yam accessions 28_TDes3033, 30_TDes3030, 31_TDesculenta, 27_TDes3035 and 29_TDes3027 were in the same group VIII ( $\mathrm{PD}=17-51)$ identified as $D$. esculenta using a reference of $D$. esculenta (KR072467) obtained from the NCBI database. In group IX (PD = 2 - 60), 86_3leavedYam-Ona, 91_TrifoliateYam-TDd, 53_Ighu, 52_Ighu-Dumenturum, 9_TDd3101, 12_TDd08-38-53, 14_TDd3100, 49_IghuUna, 84_BitterYam-Iwu-obe, 11_TDd3935, 13_TDd-yellow, 10_TDd3829 and 54_Ighu-Una-2 were found grouping with $D$. hispicia (HQ637815), D. dregeana (JQ025042) and D. dumenturum (JF705531). Group X with PD of 88 had only 62_3leavedYam-Ono and 76_Ona-TDd, while outgroups (PD = 89 - 100) contained two Ipomoea triloba (trilobed (white potatoes), Colocasia esculenta (taro) (cocoyam) and Coccinia quinqueloba (96_unknown_sample) grouped together with Solanum vermiculata and $S$. lycopersicum with NCBI accession numbers KR057204 and KM008705, respectively.

\subsection{Genetic Diversity Analysis}

The analysis involved 75 nucleotide sequences between different groups. The highest inter-group genetic distance calculated based on K2P was $5.0560 \pm$ 2.5760 , while the lowest was $0.5000 \pm 0.4770$ (Table 2). The increment in genetic diversity started from the group combinations in ascending order: $0.5000 \pm$ 0.4770 (groups, gps: I and II, I and III, I and IV, I and V) $<0.6700 \pm 0.5500$ (gps: II and VI, III and VI, V and VI) $<0.7510 \pm 0.4240$ (gps: II and IX) $<0.8100 \pm$ 0.5500 (gps: III and IX, V and IX) $<0.8820 \pm 0.5550$ (gps: I and VI) $<0.9210 \pm$ 0.4970 (gps: I and IX) $<1.0090 \pm 0.9870$ (gps: IV and VI) $<1.1390 \pm 0.9170$ (gps: IV and IX) $<1.2540 \pm 0.6540$ (gps: II and VIII) $<1.2770 \pm 0.6800$ (gps: III and VIII) $<1.3810 \pm 0.8090$ (gps: IV and VIII) $<1.5090 \pm 1.4360$ (gps: VII and VIII) $<1.5350 \pm 0.8200$ (gps: I and VIII) < $1.5820 \pm 0.8660$ (gps: VI and VIII) $<1.9060$ 
Table 2. Genetic distances based on Kimura 2-parameter (K2P) between different groups of yam species.

\begin{tabular}{|c|c|c|c|}
\hline Species 1 & Species 2 & Distance & Standard error \\
\hline Group I & Group II & 0.500 & 0.477 \\
\hline Group I & Group III & 0.500 & 0.477 \\
\hline Group I & Group IV & 0.500 & 0.477 \\
\hline Group I & Group V & 0.500 & 0.477 \\
\hline Group I & Group VI & 0.882 & 0.555 \\
\hline Group I & Group VII & 3.020 & 1.704 \\
\hline Group I & Group VIII & 1.535 & 0.820 \\
\hline Group I & Group IX & 0.921 & 0.497 \\
\hline Group II & Group III & $\mathrm{n} / \mathrm{c}$ & $\mathrm{NC}$ \\
\hline Group II & Group IV & $\mathrm{n} / \mathrm{c}$ & NC \\
\hline Group II & Group V & $\mathrm{n} / \mathrm{c}$ & $\mathrm{NC}$ \\
\hline Group II & Group VI & 0.670 & 0.550 \\
\hline Group II & Group VII & 3.020 & 1.700 \\
\hline Group II & Group VIII & 1.254 & 0.654 \\
\hline Group II & Group IX & 0.751 & 0.424 \\
\hline Group III & Group IV & $\mathrm{n} / \mathrm{c}$ & $\mathrm{NC}$ \\
\hline Group III & Group V & $\mathrm{n} / \mathrm{c}$ & $\mathrm{NC}$ \\
\hline Group III & Group VI & 0.670 & 0.550 \\
\hline Group III & Group VII & 3.020 & 1.700 \\
\hline Group III & Group VIII & 1.277 & 0.680 \\
\hline Group III & Group IX & 0.810 & 0.550 \\
\hline Group IV & Group V & $\mathrm{n} / \mathrm{c}$ & $\mathrm{NC}$ \\
\hline Group IV & Group VI & 1.009 & 0.987 \\
\hline Group IV & Group VII & 3.020 & 1.700 \\
\hline Group IV & Group VIII & 1.381 & 0.809 \\
\hline Group IV & Group IX & 1.139 & 0.917 \\
\hline Group V & Group VI & 0.670 & 0.550 \\
\hline Group V & Group VII & 3.020 & 1.700 \\
\hline Group V & Group VIII & 1.277 & 0.680 \\
\hline Group V & Group IX & 0.810 & 0.550 \\
\hline Group VI & Group VII & 5.056 & 2.576 \\
\hline Group VI & Group VIII & 1.582 & 0.866 \\
\hline Group VI & Group IX & 1.906 & 0.814 \\
\hline Group VI & Group X & 2.276 & 1.792 \\
\hline Group VII & Group VIII & 1.509 & 1.436 \\
\hline Group VII & Group IX & 3.020 & 1.676 \\
\hline
\end{tabular}


Continued

\begin{tabular}{cccc}
\hline Group IX & Group VIII & 2.456 & 1.458 \\
Group VII & Group X & 3.107 & 2.390 \\
Group IX & Group X & 1.746 & 1.479 \\
Group I & Group X & 2.718 & 2.076 \\
Group V & Group X & 2.790 & 2.130 \\
Group VIII & Group X & 1.664 & 1.491 \\
Group IV & Group X & 2.790 & 2.130 \\
Group II & Group X & 2.790 & 2.130 \\
Group III & Group X & 2.790 & 2.130 \\
\hline
\end{tabular}

Group I = 43_Gbangu_variety, 82_Yellowyam-Akpukpu, 81_Whiteyam-Nwopoko, 89_Whiteyam-Nwopoko, 24_TDm3052, 23_TDm3053, 20_TDc03-5, 19_TDc2792, 80_Utekpevariety, 17_TDc2813, 21_TDc04-71-2, 93_Yellowyam-TDes, 18_TDc2796, 68_9ENEGBE, 25_TDm3055, 15_TDc0471-2, 46_Oginivariety, 57_2-Whiteyam-Iyo, 45_Amolavariety, 40_TDa00.00.94，38_TDr89.002665，16_TDc0497-4，78_Obella, 37_Ame and 35_Pepa; Group II = 47_Damieha, 48_Aloshivariety, 39_Alata TDa98-01176,

44_Obioturuguvariety; Group III = 42_Ogojavariety; Group IV = 36_Ke-emi; Group V = 22_TDm2938; Group VI = 59_10-Whiteyam-Nwopoko-Adaka, 90_YellowYam-Oku, 33_TDaNwopoko, 41_TDa00.00600, 71_D1WaterYam-Nbana2, 1_TDa85.00250, 73_WaterYam-Mbala, 72_1WaterYam-_Nbana,

87_WaterYam-Mbana, 60_D1WaterYam-Nbana 1, 92_ChineseYam-TDes, 51_Alata2, 34_AdakavarietyIITA, and 65_YaterYam-Nbana; Group VII = 6_TDb2857, 4_TDb3050, 5_TDb3044, 83_WaterYam-_Mbana, 85_AerialYam-Edugbe, 8_TDb3690, 61_6-Edo, 3_TDa3050 and TDb3058; Group VIII = 28_TDes3033, 30_TDes3030, 31_TDesculenta, 27_TDes3035 and 29_TDes3027; Group IX = 86_3leavedYam-Ona, 91_TrifoliateYam-TDd, 53_Ighu, 52_Ighu-Dumenturum, 9_TDd3101, 12_TDd08-38-53, 14_TDd3100, 49_IghuUna, 84_BitterYam-Iwu-obe, 11_TDd3935, 13_TDd-yellow, 10_TDd3829 and 54_Ighu-Una-2; and Group X = 62_3leavedYam-Ono and 76_Ona-TDd, N/C = Not computable.

\pm 0.8140 (gps: VI and IX) $<1.6640 \pm 1.4910$ (gps: VIII and X) $<1.7460 \pm 1.4790$ (gps: $2.2760 \pm 1.7920$ (gps: VI and X) < $2.4560 \pm 1.4580$ (gps: VIII and IX) $<$ $2.7180 \pm 2.0760$ (gps: I and X) $<2.7900 \pm 2.1300$ (gps: II and X, III and X, IV and $\mathrm{X}, \mathrm{V}$ and $\mathrm{X}$ ) $<3.0200 \pm 1.7000$ (gps: I and VII, II and VII, III and VII, IV and VII, V and VII, VII and IX) $<3.1070 \pm 2.390$ (gps: VII and X) $<5.0560 \pm 2.5760$ (gps: VI and VII). The intra-group genetic distance ranged from $0.5250 \pm 0.5000$ - $2.0103 \pm 1.2579$ and some intra-group genetic distances were not computable which were denoted by $\mathrm{n} / \mathrm{c}$ (Table 3 ). Groups I, VI and X were found computable with their respective values of $0.5250 \pm 0.5000,0.5616 \pm 0.4788$, and 2.0103 \pm 1.2579 . The mean genetic diversity within entire population was $0.7970 \pm$ 0.06910 , while the transitional to transversional distances per site from mean interpopulational diversity calculations was $2.1478 \times 10^{8} \pm 4.5300$. Also, the coefficient of differentiation of transitional to transversional distances per site was $1.1947 \times 10^{8} \pm 6.9419 \times 10^{7}$.

\subsection{BLAST Analysis of the Sequences Generated from the Yam Accessions Using rbcL Barcoding Gene}

The output of the BLAST computations of the grouped sequences produced significant hits and some of the previously unknown sequences were fully identified (Table 4). The analysis identified ten putative species of yams including Dioscorea alata, $D$. bulbifera, $D$. cayenensis, $D$. rotundata, $D$. wallichii, D. aspersa, 
Table 3. Genetic distances based on Kimura 2-parameter (K2P) within different groups of yam species.

\begin{tabular}{|c|c|c|}
\hline Group name & Distance & Standard error \\
\hline Group I & 0.5250 & 0.5000 \\
\hline Group II & $\mathrm{n} / \mathrm{c}$ & $\mathrm{n} / \mathrm{c}$ \\
\hline Group III & $\mathrm{n} / \mathrm{c}$ & $\mathrm{n} / \mathrm{c}$ \\
\hline Group IV & $\mathrm{n} / \mathrm{c}$ & $\mathrm{n} / \mathrm{c}$ \\
\hline Group V & $\mathrm{n} / \mathrm{c}$ & $\mathrm{n} / \mathrm{c}$ \\
\hline Group VI & 0.5616 & 0.4788 \\
\hline Group VII & $\mathrm{n} / \mathrm{c}$ & $\mathrm{n} / \mathrm{c}$ \\
\hline Group II & $\mathrm{n} / \mathrm{c}$ & $\mathrm{n} / \mathrm{c}$ \\
\hline Group III & $\mathrm{n} / \mathrm{c}$ & $\mathrm{n} / \mathrm{c}$ \\
\hline Group X & 2.0103 & 1.2579 \\
\hline
\end{tabular}

Group I = 43_Gbangu_variety, 82_Yellowyam-Akpukpu, 81_Whiteyam-Nwopoko, 89_Whiteyam-Nwopoko, 24_TDm3052, 23_TDm3053, 20_TDc03-5, 19_TDc2792, 80_Utekpevariety, 17_TDc2813, 21_TDc04-71-2, 93_Yellowyam-TDes, 18_TDc2796, 68_9ENEGBE, 25_TDm3055, 15_TDc0471-2, 46_Oginivariety, 57_2-Whiteyam-Iyo, 45_Amolavariety, 40_TDa00.00.94, 38_TDr89.002665, 16_TDc0497-4, 78_Obella, 37_Ame and 35_Pepa; Group II = 47_Damieha, 48_Aloshivariety, 39_Alata TDa98-01176, 44_Obioturuguvariety; Group III = 42_Ogojavariety; Group IV = 36_Ke-emi; Group V = 22_TDm2938; Group VI = 59_10-Whiteyam-Nwopoko-Adaka, 90_YellowYam-Oku, 33_TDaNwopoko, 41_TDa00.00600, 71_D1WaterYam-Nbana2, 1_TDa85.00250, 73_WaterYam-Mbala, 72_1WaterYam-_Nbana, 87_WaterYam-Mbana, 60_D1WaterYam-Nbana 1, 92_ChineseYam-TDes, 51_Alata2, 34_AdakavarietyIITA, and 65_YaterYam-Nbana; Group VII = 6_TDb2857, 4_TDb3050, 5_TDb3044, 83_WaterYam-_Mbana, 85_AerialYam-Edugbe, 8_TDb3690, 61_6-Edo, 3_TDa3050 and TDb3058; Group VIII = 28_TDes3033, 30_TDes3030, 31_TDesculenta, 27_TDes3035 and 29_TDes3027; Group IX = 86_3leavedYam-Ona, 91_TrifoliateYam-TDd, 53_Ighu, 52_Ighu-Dumenturum, 9_TDd3101, 12_TDd08-38-53, 14_TDd3100, 49_IghuUna, 84_BitterYam-Iwu-obe, 11_TDd3935, 13_TDd-yellow, 10_TDd3829 and 54_Ighu-Una-2; and Group X = 62_3leavedYam-Ono and 76_Ona-TDd.

Table 4. BLAST outputs of total score, query coverage, e-value, percentage identity and accession number obtained from different yam accessions.

\begin{tabular}{|c|c|c|c|c|c|c|}
\hline Sequence name & Hit in NCBI database & Total score & Query coverage & E-value & \%Identity & Accession No \\
\hline 1_TDa85.00250 & Dioscorea alata & 852 & 852 & 0 & 100 & KY710782 \\
\hline 3_TDa3050 & D. bulbifera & 736 & 736 & 0 & 100 & KR087030 \\
\hline 4_TDb3050 & D. bulbifera & 771 & 100 & 0 & 100 & KR087030 \\
\hline 5_TDb3044 & D. bulbifera & 756 & 100 & 0 & 100 & KR087030 \\
\hline 6_TDb2857 & D. bulbifera & 839 & 100 & 0 & 100 & KR087030 \\
\hline 7_TDb3058 & D. bulbifera & 826 & 100 & 0 & 99 & KR087030 \\
\hline 8_TDb3690 & D. bulbifera & 737 & 100 & 0 & 100 & KR087030 \\
\hline 9_TDd3101 & D. dregeana & 1009 & 100 & 0 & 100 & KR087039 \\
\hline 10_TDd3829 & D. dregeana & 985 & 100 & 0 & 100 & KR087039.1 \\
\hline 11_TDd3935 & D. dregeana & 996 & 100 & 0 & 100 & KR087039.1 \\
\hline 12_TDd08-38-53 & D. dregeana & 1005 & 100 & 0 & 100 & KR087039.1 \\
\hline 13_TDdYellow & D. dregeana & 996 & 100 & 0 & 100 & KR087039.1 \\
\hline 14_TDd3100 & D. dregeana & 1003 & 100 & 0 & 100 & KR087039.1 \\
\hline 15_TDc04-71-2 & D. wallichii & 835 & 100 & 0 & 100 & MF142259.1 \\
\hline 16_TDc04-97-4 & D. cayenensis/rotundata & 1005 & 100 & 0 & 100 & $\begin{array}{c}\text { KJ629254.1/ } \\
\text { KJ490011.1 }\end{array}$ \\
\hline 17_TDc2813 & D. rotundata & 743 & 100 & 0 & 100 & KY679568.1 \\
\hline
\end{tabular}


G. N. Ude et al.

\section{Continued}

\begin{tabular}{|c|c|c|c|c|c|c|}
\hline 18_TDc2796 & D. rotundata & 715 & 100 & 0 & 100 & KY679568.1 \\
\hline 19_TDc2792 & D. wallichiil rotundata & 739 & 100 & 0 & 100 & $\begin{array}{c}\text { MF142259.1/ } \\
\text { KY679568.1 }\end{array}$ \\
\hline 20_TDc03-5 & D. rotundata & 739 & 100 & 0 & 100 & KY679568.1 \\
\hline 21_TDc04-71-2 & D. rotundata & 758 & 100 & 0 & 100 & KY679568.1 \\
\hline 22_TDm2938 & D. cayenensis/rotundata & 1002 & 100 & 0 & 100 & $\begin{array}{c}\text { KJ629254.1/ } \\
\text { KJ490011.1 }\end{array}$ \\
\hline 23_TDm3053 & D. rotundata & 739 & 100 & 0 & 100 & KY679568.1 \\
\hline 24_TDm3052 & D. rotundata & 739 & 100 & 0 & 100 & KY679568.1 \\
\hline 25_TDm3055 & D. wallichiil rotundata & 824 & 100 & 0 & 100 & $\begin{array}{c}\text { MF142259.1/ } \\
\text { KY679568.1 }\end{array}$ \\
\hline 27_TDes3035 & D. esculenta & 941 & 100 & 0 & 100 & KJ956696.1 \\
\hline 28_TDes3033 & D. esculenta & 828 & 100 & 0 & 100 & KJ956696.1 \\
\hline 29_TDes3027 & D. esculenta & 998 & 100 & 0 & 100 & KJ956696.1 \\
\hline 30_TDes3030 & D. esculenta & 736 & 100 & 0 & 100 & KJ956696.1 \\
\hline 31_TDesculenta & D. esculenta & 734 & 100 & 0 & 100 & KJ956696.1 \\
\hline 33_TDaNwopoko & D. alata & 1003 & 100 & 0 & 100 & KY710782.1 \\
\hline 34_Adakavariety.IITA & D. alata & 1000 & 100 & 0 & 100 & KY710782.1 \\
\hline 35_Pepa & D. cayenensis/rotundata & 1003 & 100 & 0 & 100 & $\begin{array}{c}\text { KJ629254.1/ } \\
\text { KJ490011.1 }\end{array}$ \\
\hline 36_Ke-emi & D. cayenensis/rotundata & 981 & 100 & 0 & 100 & $\begin{array}{c}\text { KJ629254.1/ } \\
\text { KJ490011.1 }\end{array}$ \\
\hline 37_Ame & D. cayenensis/ rotundata & 1000 & 100 & 0 & 100 & $\begin{array}{c}\text { KJ629254.1/ } \\
\text { KJ490011.1 }\end{array}$ \\
\hline 38_TDr89.002665 & D. cayenensis/rotundata & 1005 & 100 & 0 & 100 & $\begin{array}{c}\text { KJ629254.1/ } \\
\text { KJ490011.1 }\end{array}$ \\
\hline 39_AlataTda_98.01176 & D. cayenensis/rotundata & 1007 & 100 & 0 & 100 & $\begin{array}{l}\text { KJ629254.1/ } \\
\text { KJ490011.1 }\end{array}$ \\
\hline 40_TDa00.00.94 & D. cayenensis/rotundata & 1005 & 100 & 0 & 100 & $\begin{array}{c}\text { KJ629254.1/ } \\
\text { KJ490011.1 }\end{array}$ \\
\hline 41_Tda00.00600 & D. rotundata & 992 & 100 & 0 & 100 & KY710782.1 \\
\hline 42_OgojaVariety.1 & D. cayenensis/rotundata & 998 & 100 & 0 & 100 & $\begin{array}{c}\text { KJ629254.1/ } \\
\text { KJ490011.1 }\end{array}$ \\
\hline 43_GbanguVariety.1 & $\begin{array}{l}\text { D. praehensilis/ cayennensis/ } \\
\text { rotundata }\end{array}$ & 955 & 100 & 0 & 99 & $\begin{array}{c}\text { KR072476.1/ } \\
\text { KJ629254.1/ } \\
\text { KJ490011.1 }\end{array}$ \\
\hline 44_ObioturuguVariety.1 & D. cayenensis/rotundata & 1011 & 100 & 0 & 100 & $\begin{array}{l}\text { KJ629254.1/ } \\
\text { KJ490011.1 }\end{array}$ \\
\hline 45_AmolaVariety.1 & D. cayenensis/rotundata & 1005 & 100 & 0 & 100 & $\begin{array}{l}\text { KJ629254.1/ } \\
\text { KJ490011.1 }\end{array}$ \\
\hline 46_OginiVariety & D. wallichiil rotundata & 857 & 100 & 0 & 100 & $\begin{array}{c}\text { MF142259.1/ } \\
\text { KY679568.1 }\end{array}$ \\
\hline 47_Damieha & D. cayenensis/rotundata & 1005 & 100 & 0 & 100 & $\begin{array}{c}\text { KJ629254.1/ } \\
\text { KJ490011.1 }\end{array}$ \\
\hline
\end{tabular}




\section{Continued}

\begin{tabular}{|c|c|c|c|c|c|c|}
\hline 48_Aloshivariety.1 & D. cayenensis/rotundata & 1007 & 100 & 0 & 100 & $\begin{array}{l}\text { KJ629254.1/ } \\
\text { KJ490011.1 }\end{array}$ \\
\hline 49_IghuUna & D. dregeanal hispida & 992 & 100 & 0 & 99 & $\begin{array}{l}\text { KR087039/ } \\
\text { HQ637815.1 }\end{array}$ \\
\hline 51_Alata.2 & D. alata & 736 & 100 & 0 & 100 & KY710782.1 \\
\hline 52_Ighu_Dumenturum & D. hispida & 774 & 100 & 0 & 100 & KY710783.1 \\
\hline 53_Ighu & D. dumetorum/ hispida & 830 & 100 & 0 & 100 & KY710783.1 \\
\hline 54_IghuUna.2 & D. hispidal dumetorum & 756 & 100 & 0 & 100 & KY710783.1 \\
\hline 57_2-WhiteYam-_Iyo & D. rotundata & 872 & 100 & 0 & 100 & KR072483.1 \\
\hline 59_10-WhiteNwopoko-Adaka & $\begin{array}{l}\text { D. spicatal intermedial wallichiil } \\
\text { rotundatal oppositiflia }\end{array}$ & 534 & 100 & $4.00 \mathrm{E}-148$ & 100 & $\begin{array}{c}\text { KY457460.1/ } \\
\text { KY457459.1/ } \\
\text { KY679569.1/ } \\
\text { KY679568.1/ } \\
\text { KY679566.1 }\end{array}$ \\
\hline 60_D1Water-Nbana1 & D. alata & 778 & 100 & 0 & 100 & KY710782.1 \\
\hline 61_6-EDO & D. bulbifera & 737 & 100 & 0 & 100 & KR087030.1 \\
\hline 62_3LeavedYam-Ono & D. aspersal petelotiil daunea & 665 & 99 & 0 & 97 & $\begin{array}{r}\text { HQ637816.1/ } \\
\text { AY904802.1/ } \\
\text { AY904793.1 }\end{array}$ \\
\hline 65_WaterYam.Nbana & D. alata & 730 & 100 & 0 & 100 & KY710782.1 \\
\hline 68_9ENEGBE & D. rotundata & 822 & 100 & 0 & 100 & KR072483.1 \\
\hline 71_D1WaterYam-Nbana2 & D. alata & 989 & 100 & 0 & 100 & KY710782.1 \\
\hline 72_1-WaterYam-_Nbana & D. alata & 798 & 100 & 0 & 100 & KY710782.1 \\
\hline 73_Wateryamji_mbala & D. alata & 852 & 100 & 0 & 100 & KY710782.1 \\
\hline 76_OnaTDd & D. aspersa & 612 & 100 & $2.00 \mathrm{E}-171$ & 97 & HQ637816.1 \\
\hline 78_Obella & D. cayenensis/rotundata & 992 & 100 & 0 & 100 & $\begin{array}{c}\text { KJ629254.1/ } \\
\text { KJ490011.1 }\end{array}$ \\
\hline 80_UtekpeVariety_2 & D. wallichiil rotundata & 741 & 100 & 0 & 100 & $\begin{array}{r}\text { MF142259.1/ } \\
\text { KY679568.1 }\end{array}$ \\
\hline 81_WhiteYam-Nwoopoko & D. rotundata & 737 & 100 & 0 & 100 & KR072483.1 \\
\hline 82_Yellowyam_Akpukpu & D. rotundata & 806 & 100 & 0 & 100 & KR072483.1 \\
\hline 83_WaterYam-_Mbuna & D. bulbifera & 750 & 100 & 0 & 100 & KR087030.1 \\
\hline 84_BitterYam-Iwu_obe & D. dregeanal hispida & 998 & 100 & 0 & 100 & $\begin{array}{c}\text { KR087039/ } \\
\text { HQ637815.1 }\end{array}$ \\
\hline 85_AerialYam_Edugbe & D. bulbifera & 739 & 100 & 0 & 100 & KR087030.1 \\
\hline 86_3LeavedYam_Ona & D. hispida & 861 & 100 & 0 & 100 & KU865503.1 \\
\hline 87_WaterYam-Mbana & D. alata & 782 & 100 & 0 & 100 & KY710782.1 \\
\hline 89_WhiteYam_Nwoopoko & D. rotundata & 739 & 100 & 0 & 100 & KR072483.1 \\
\hline 90_Yellowyam_Oku & D. alata & 963 & 100 & 0 & 100 & KY710782.1 \\
\hline 91_TrifoliateYam_TDd & D. hispida & 837 & 100 & 0 & 100 & KU865503.1 \\
\hline 92_ChineseYam_TDes & D. alata & 739 & 100 & 0 & 100 & KY710782.1 \\
\hline 93_YellowYam_TDes & D. rotundata & 697 & 100 & 0 & 100 & KY710782.1 \\
\hline
\end{tabular}


$D$. trifida, D. dregeana, and $D$. mangenotiana. The total bit score obtained in all ranged from 411 - 1011. The query coverage spanned between 99 and 100\%, while the expected values (e-values) were $9 \mathrm{e}^{-111}$ or less. The percentage sequence identity ranged from $97 \%-100 \%$. Some accessions with acronyms including TDa, TDc and TDm denoting D. alata, D. cayenensis and D. manganotiana were found to be D. bulbifera, D. rotundata or cayenensis, respectively. Some of the sequences had NCBI hits ranging from two to four sequences with synonymous values of total bit score, query coverage, e-value, percentage identity but different accession numbers. For instance, 16_TDc04-97-4, 22_TDm2938, 35_Pepa, 36_Ke-emi, 37_Ame, 38_TDr.89.002665 and many others in this category had hits of $D$. cayenensis and D. rotundata. For accessions of 19_TDc2792, 25_TDm3055, 46_OginiVariety and 80_UtekpeVariety_2 had $D$. wallichii and $D$. rotundata as their hits with similar values in all the BLAST indices. Also, three species of yam including $D$. praehensilis, $D$. cayenensis and $D$. rotundata were obtained with a yam accession of 43_Gbangu_Variety.1 in the process of BLAST analysis, while 62_3LeavedYam-Ono produced $D$. aspersa, $D$. petelotii and $D$. daunea that had same values of total bit score, query coverage, e-value, percentage identity but different accession numbers. The yam accession, 59_D10 White-Nwopoko-Adaka, had five different NCBI hits of D. spicata, D. intermedia, D. wallichii, D. rotundata and D. oppositifolia with three having similar accession number, while the remaining two had a separate accession number as revealed by BLAST analysis.

\section{Discussion}

DNA barcoding has become an effective method for species discrimination of flowering plants in the Polygonaceae [35] [47] and Fabaceae families [39], and other land plant species [35] [42] [48] [49]. While mitochondrial cytochrome oxidase 1 (CO1) has proven a standardized animal DNA barcoding for necessary discrimination, no single barcode sequence works across all plants [49]. In the present work, the candidate barcoding marker, rbcL satisfied the DNA barcoding process, regarding the ease of amplification and sequencing Hollingsworth et al. [49]. However, this barcoding marker, rbcL, was not able to achieve the basic quality of discriminating different yam species in this study. Sequence alignment showed low degree of polymorphisms among the sequences. This study of genetic diversity in yam accessions is also dependent on the nucleotide variations occurring within the genome that are informative for the identification of different species. The discriminatory level of the rbcL marker has been linked to other researches, which contradict its potential for use as a universal DNA barcode for plants [50] [51] [52]. This low resolution of different accessions of yams into their respective species level could be attributed to the poor efficiency of rbcL marker when not jointly applied with other plastid markers. It has been reported that the joint application of rbcL+matK as a marker of choice in species resolution was based on clear recovery of the region of $\mathrm{rbcL}$ and discriminatory efficiency of fast evolving coding region of matK [53]. 
In this study, 525 bp distinct total lengths of sequence alignment, 534 conserved sites, and variable sites of 7 were identified in the sequenced yam species. The alignment of $525 \mathrm{bp}$ out of the total lengths of $568 \mathrm{bp}$, followed by the existence of similar regions (conserved sites) and low points of variations (variable areas) among the sequences demonstrate the low level of informativeness of rbcL in DNA barcoding of yam species. These findings are not in complete agreement with a previous report on yam species [30], where $568 \mathrm{bp}, 538$, and 30 as total lengths of sequence alignment, conserved sites and variable sites were identified among accessions. Also, the sequence alignment length, conserved sites excluding the variable sites detected in this work correlate with the findings of Sun et al. [54] in which $553 \mathrm{bp}, 522 \mathrm{bp}$ and 31 of alignment length, conserved sites and variable sites were found among the accessions of Dioscorea species. The difference in the variable sites could have emanated from the number of samples studied.

Phylogenetic reconstruction of the generated Dioscorea species using rbcL marker resolved them into ten groups and this indicates different existing isolated groups inherent in the accessions. The existence of these different accessions among the collections could be attributable to lack of exchange of yam tubers by farmers among villages thereby resulting in a stronger heterozygosity among species compared to wild ones as reported by Ngo Ngwe et al. [24]. A contribution of evolutionary biology regarding conservation is the knowledge of diverse phylogenetic diversities, defined by the sums of branch lengths of the evolutionary trees connecting a set of taxa or individuals [55]. In this present work, group X had the highest PD value of 88 , followed by groups VII, VI, IX, and VIII with their respective PDs of 86, 79, 60 and 51. The highest PD was identified in a group containing wild species of $D$. aspersa and this is in agreement with a previous report though in a different wild species wild called $D$. praehensilis [24]. When compared with other unrelated crops, the highest was observed in Cocoyam and other crops which were deliberately included to access the accuracy of this marker. The group with the lowest PD value D. rotundata clustered with other species and they were collected from a given single region. In this way, a given set of taxa will have a greater PD if they are widely spread out on a phylogenetic tree. Lack of or total loss of PD is generally assumed as a declining signal in the degree of biodiversity [56]. Furthermore, PD is associated with functional diversity since it is a measure of features also due to the fact that evolutionarily distant species are more likely to possess variable molecular functions in an ecosystem [28] [57] [58]. Also in group I, some accessions including 45_Amolavariety, 40_TDa00.00.94, 38_TDr89.002665, 16_TDc0497.4, 78_Obella, 37_Ame, and 35_Pepa had a PD value of 0 and this could be attributed to lack of sequence divergence. It could also be attributable to occurrence of common ancestral sequence homology [59] or poor resolving power of the rbcL DNA barcoding marker in yams [54]. Most of the accessions were accurately grouped according their species. For instance in group VIII, all the D. esculenta species was 
grouped with a known reference sequence from NCBI database. Also, group VII had all the accessions classified as $D$. bulbifera thereby identifying correctly an accession, 3_TDa3050, which was regarded as D. alata. A particular yam species was given different names as Ighu or Una (Ona) at a village in Enugu State but it was found to be just one species called $D$. dumetorum through the use of rbcL thereby resolving the issue of multiple names for the plant. Group IX had three reference sequences as $D$. dregeana, D. hispida and D. dumetorum but most of the accessions in the group are D. dumetorum and this could be as a result of their genetic relatedness [59]. However, accessions in group I of the trees were not correctly resolved following the existence of different yam species in various distinct subclades and non-grouping of any of the retrieved yam sequences from NCBI database. This may possibly be linked to sample contamination or a deficiency on the part of the rbcL resolution.

The identified genetic distances $(0.5000 \pm 0.4770-5.0560 \pm 2.5760)$ based on $\mathrm{K} 2 \mathrm{P}$ model regarding the inter-groups were in agreement with the previous works of other researchers in yams [24] [54] and in authentication of native plants [60]. High genetic diversity indices were obtained from between group calculations, producing $5.0560 \pm 2.5760$ with the highest in two combined groups (groups VI and VII) and this demonstrates higher interspecific diversity than intraspecific one within the yam accessions as obtained in an earlier report involving ornamental plants with interspecific value of 3.080 [61]. Assessment of genetic diversity within the groups (intra-group genetic diversity) could not be computed in most of the groups except three groups (groups I, VI and X), where group I had the lowest value of $0.5250 \pm 0.5000$, while $\mathrm{X}$ had the highest value of $2.0103 \pm 1.2579$. These values are higher than the ones obtained by Sun et al. [54]. The mean genetic diversity within entire population was $0.7970 \pm 0.06910$ and this is higher than the one $(0.00266 \pm 0.0044)$ obtained by Sun et al. [54].

BLAST hits obtained in this study showed some degrees of similarity matches to the ones already annotated and deposited in NCBI database and some were not purely specific. The percentage sequence identity ranged from $97 \%-100 \%$, demonstrating low efficiency of this tool in identification of unknowns in yam species. However, some of the yams sampled from different regions were differently identified from what they were previously known to be using this method, indicating the potential of rbcL barcoding marker to resolve misclassification encountered via morphotaxonomy based approach despite the low discriminatory power. For instance, yam accessions with acronyms including TDa, TDc and TDm denoting D. alata, D. cayenensis and D. manganotiana were found to be $D$. bulbifera, $D$. rotundata or cayenensis, respectively. Furthermore, 62_3LeavedYam-Ono and 76_Ona_TDd sequences were correctly identified as $D$. aspersa. In the community where the two species (D. aspersa and D. dumetorum) were collected, they were misclassified by the villagers who generally called them $D$. dumetorum due to their similar morphological features. According to the villagers, the ones in group $\mathrm{X}$ which were later identified as $D$. aspersa are normally boiled and eaten directly, while the other ones ( $D$. dumetorum, 
which had similar values of NCBI hits with $D$. hispida) are usually boiled, processed to remove bitterness in them before they are consumed. The discriminatory level of the rbcL marker in plants as a potential universal DNA barcode is demonstrated in this study as reported in other researches [50] [51]. However, some of the yam sequences had two, three or five NCBI hits of different species of yams with synonymous values of total bit score, query coverage, -value and percentage identity with different accession numbers except in one that had five BLAST outputs with three having similar accession numbers and two with different accession numbers. For instance, the yam accession, 59_D-10-WhiteNwopoko-Adaka, had five different NCBI hits of D. spicata, D. intermedia, $D$. wallichii, D. rotundata and D. oppositifolia with three (D. wallichii, D. rotundata, D. oppositifolia) having similar accession number (KY679569), while the remaining two (D. spicata and D. intermedia) had separate accession numbers of KY457460 and KY457459, respectively, after the BLAST analysis. Also, sequences generated from accessions 45_Amolavariety, 40_TDa00.00.94, 38_TDr89.002665, 16_TDc0497.4, 78_Obella, 37_Ame, and 35_Pepa hit two ( $D$. cayenensis and $D$. rotundata) sequences with similar values of query coverage, e-value and percentage identity, while total bit score ranged from 1000-1005. This is possible due to existence of common ancestral homology as opined by Pearson [59] or due to redundancy, which in bioinformatics is observed when one or more homologous or synonymous sequences are found in the same set of data [62]. It could also be attributable to the low discriminatory potency of rbcL marker to correctly resolve species as previously reported in yams [54] and ornamental plants [61].

\section{Conclusion}

The candidate barcoding marker, rbcL, was found to be ambiguously discriminatory in DNA barcoding process of yam accessions. Some of the accessions were not correctly identified to the species level and low polymorphisms were detected and this further demonstrates the low distinguishing potency of rbcL barcoding marker. The use of phylogenetic diversity (PD), which is associated with functionality in biodiversity and which was applied in the computational processes for the estimation of phylogenetic groups with lowest and largest collections in terms of diversity was of great potential. The highest phylogenetic diversity was in $D$. aspersa, while some were not computable due to the low efficacy of the marker. The group with the lowest PD value, $D$. rotundata clustered with other indistinguishable species and they were collected from a given single region. The accessions with high PD within the yam accessions should be considered for use in breeding programme to enhance biodiversity of Dioscorea species within the studied region. However, the rbcL could not resolve the yam accessions well following some noted discrepancies in the detected number of species from phylogenetic groupings and NCBI BLAST hits possibly due to inefficiency of the marker. Therefore, the rbcL may not be a marker of choice for species identification, discrimination and estimation of genetic diversity of yam 
accessions. The marker should be used in combination with other chloroplast markers for accurate DNA barcoding of yams for their improvement and germplasm conservation.

\section{Acknowledgements}

The authors are grateful to International Institute of Tropical Agriculture (IITA), Ibadan for providing part of the accessions used in the study. We thank National Science Foundation (NSF) for the Targeted Infusion HBCU_UP funding that supported this undergraduate student's research project. We are also grateful to Dr. Dave Micklos of the Cold Spring Harbor Laboratory, DNA Learning Centre, for the technical and research assistance offered to us.

\section{Funding}

National Science Foundation (NSF) for the Targeted Infusion HBCU_UP funding was received to conduct this study

\section{Ethics Approval and Consent to Participate}

Consent was obtained from farmers before using their individual farms for sample collection.

\section{Consent for Publication}

Not applicable.

\section{Availability of Data and Materials}

All data generated during this study are included in this published article. Sequence data were deposited in NCBI GenBank with accession numbers ranging from MH078114 to MH078188 to match the individual yam accessions in the list of supplementary Table S1.

\section{Authors' Contributions}

All authors were involved in project design. GNU, DOI, JM, OO, JH, DB, CA and $\mathrm{OC}$ did the literature search process, extracted data elements, and carried out study compilation. Data analyses were performed by DOI, MO, CE, VC, MU and $\mathrm{CO}$ and reviewed by GNU, GA, JO and AD. DOI developed the first draft of the manuscript. All authors read the manuscript and approved the final copy of it.

\section{Conflicts of Interest}

The authors declare that they have no competing interests.

\section{References}

[1] Wilkin, P., Schols, P., Chase, M.W., Chayamarit, K., Furness, C.A., Huysmans, S., Rakotonasolo, F., Smets, E., Thapyai, C. and Meerow, A.W. (2005) A Plastid Gene Phylogeny of the Yam Genus, Dioscorea: Roots, Fruits and Madagascar. Systematic 
Botany, 30, 736-749. https://doi.org/10.1600/036364405775097879

[2] Govaerts, R., Wilkin, P. and Saunders, R.M.K. (2007) World Checklist of Dioscoreales. Yams and Their Allies. Kew Publisher, Royal Botanic Gardens, Kew.

[3] Norman, P.E., Tongoona, P. and Shanahan, P.E. (2011) Diversity of the Morphological Traits of Yam (Dioscorea spp.) Genotypes from Sierra Leone. Journal of Applied Bioscience, 45, 3045-3058.

[4] Scarcelli, N., Tostain, S., Mariac, C., Agbangla, C., Ogoubi, D., Julien, B. and Pharm, J.L. (2006) Genetic Nature of Yams (Dioscorea sp.) Domesticated by Farmers in Benin (West Africa). Genetic Resource and Crop Evolution, 53, 121-130. https://doi.org/10.1007/s10722-004-1950-5

[5] Lebot, V. (2009) Tropical Root and Tuber Crops: Cassava, Sweet Potato, Yams and Aroids. CABI Publishers, Wallingford, UK.

[6] FAO (2012) Food and Agricultural Organization. FAOSTATDATA. FAO, Rome. http://faostat.fao.org/

[7] Arnau, G., Abraham, K., Sheela, M. N., Chair, H., Sartie, A. and Asiedu, R. (2010) Yams. In: Bradshaw, J.E., Ed., Root and Tuber Crops, Springer, New York, 127-148. https://doi.org/10.1007/978-0-387-92765-7_4

[8] Hou, W.C., Hsu, F.L. and Lee, M.H. (2002) Yam (Dioscorea batatas) Tuber Mucilage Antioxidant Activities in-Vitro. Planta Medica, 68, 1072-1076.

https://doi.org/10.1055/s-2002-36356

[9] Nweke, F.I., Ugwu, B.O., Asadu, C.L.A. and Ay, P. (1991) Production Costs in the Yam-Based Cropping Systems of South-Western Nigeria. Resource and Crop Management Division. Research Monograph No 6, IITA Ibadan, 29 p.

[10] Degras, L. (1993) The Yam, a Tropical Root Crop. London, 40 p.

[11] Asiedu, R. and Sartie, A. (2010) Crops That Feed the World Yams. Food Security, 2, 305-315. https://doi.org/10.1007/s12571-010-0085-0

[12] Zhou, Y., Zhou, C., Yao, H., Liu, Y. and Tu, R. (2008) Application of ISSR Markers in Detection of Genetic Variation among Chinese Yam (Dioscorea opposita Thunb) Cultivars. Life Science Journal, 5, 4.

[13] Djeri, B., Tchobo, P.F., Adjrah, Y., Karou, D.S., Ameyapoh, Y., Soumanou, M.M. and Souza, C. (2015) Nutritional Potential of Yam Chips (Dioscorea cayenensis and Dioscorea rotundata Poir) Obtained Using Two Methods of Production in Togo. African Journal of Food Science, 9, 278-284. https://doi.org/10.5897/AJFS2014.1207

[14] Mahalakshmi, V., Ng, Q. and Atalobhor, K. (2007) Development of West African Yam Dioscorea spp. Core Collection. Genetic Resource and Crop Evolution, 54, 1817-1825. https://doi.org/10.1007/s10722-006-9203-4

[15] Kenyon, L., Lebas, B.S.M. and Seal, S.E. (2008) Yams (Dioscorea spp.) from the South Pacific Islands Contain Many Novel Badnaviruses: Implications for International Movement of Yam Germplasm. Archives of Virology, 153, 877-889. https://doi.org/10.1007/s00705-008-0062-5

[16] IITA (2009) ITTA Annual Report. International Institute of Tropical Agriculture, Ibadan.

[17] FAO (2011) Food and Agricultural Organization. FAO, Rome. http://faostat.fao.org/

[18] Mignouna, H.D., Dansi, A. and Zok, S. (2002) Morphological and Isozymic Diversity of the Cultivated Yams (Dioscorea cayenensis/Rotundata Complex) of Cameroun. Genetic Resource and Crop Evolution, 49, 21-29. 
https://doi.org/10.1023/A:1013805813522

[19] Schwenk, K., Brede, N. and Streit, B. (2008) Introduction. Extent, Processes and Evolutionary Impact of Interspecific Hybridization in Animals. Philosophical Transactions of the Royal Society B, 363, 2805-2811.

[20] Paun, O., Forest, F., Fay, M.F. and Chase, M.W. (2009) Hybrid Speciation in Angiosperms: Parental Divergence Drives Ploidy. New Phytologist, 182, 507-518. https://doi.org/10.1111/j.1469-8137.2009.02767.x

[21] Whitney, K.D., Ahern, J.R., Campbell, L.G., Albert, L.P. and King, M.S. (2010) Patterns of Hybridization in Plants. Perspectives in Plant Ecology, 12, 175-182.

[22] Primack, R.B. (2012) A Primer of Conservation Biology. 5th Edition, Edition Sinauer Assiociates, Sunderland, 363 p.

[23] Dansi, A., Orobiyi, A., Dansi, M., Assogba, P., Sanni, A. and Akpagana, K. (2013) Sélection de sites pour la conservation in situ des ignames sauvages apparentées aux ignames Cultivées: Cas de Dioscorea praehensilis Au Bénin. International Journal of Biological and Chemical Sciences, 7, 60-74. https://doi.org/10.4314/ijbcs.v7i1.6

[24] Ngo Ngwe, M.F.S., Omokolo, D.N. and Joly, S. (2015) Evolution and Phylogenetic Diversity of Yam Species (Dioscorea spp.): Implication for Conservation and Agricultural Practices. PLoS ONE, 10, e0145364. https://doi.org/10.1371/journal.pone.0145364

[25] Purvis, A., Gittleman, L. and Books, J.T. (2005) Phylogeny and Conservation. Cambridge University Press, London, 120-138. https://doi.org/10.1017/CBO9780511614927

[26] Winter, M., Schweiger, O., Klotz, S., Nentwig, W., Andriopoulos, P., Arianoutsou, M., Basnou, C., Delipetrou, P., Didziulis, V., Hejda, M., Hulm, P.E., Lambdon, P.W., Perglh, J., Pysek, P., Royl, D.B. and Kuhn, I. (2009) Plant Extinctions and Introductions Lead to Phylogenetic and Taxonomic Homogenization of the European Flora. Proceeding of National Academy of Science, 106, 21721-21725. https://doi.org/10.1073/pnas.0907088106

[27] Faith, D.P., Magallón, S., Hendry, A.P., Conti, E., Yahara, T. and Donoghue, M.J. (2010) Ecosystem Services: An Evolutionary Perspective on the Links between Biodiversity and Human Well-Being. Current Opinion in Environmental Sustainability, 2, 66-74. https://doi.org/10.1016/j.cosust.2010.04.002

[28] Srivastava, D.S. and Vellend, M. (2005) Biodiversity-Ecosystem Function Research: Is It Relevant to Conservation? Annual Review of Ecology and Evolution System, 36, 267-294. https://doi.org/10.1146/annurev.ecolsys.36.102003.152636

[29] Tamiru, M., Becker, H.C. and Maass, B.L. (2011) Comparative Analysis of Morphological and Farmers Cognitive Diversity in Yam Landraces (Dioscorea spp.) from Sothern Ethiopia. Tropical Agriculture and Development, 55, 28-43.

[30] Girma, G., Spillane, C. and Gedil, M. (2015) DNA Barcoding of the Main Cultivated Yams and Selected Wild Species in the Genus Dioscorea. Journal of Systematic and Evolution, 9999, 1-10.

[31] Terauchi, R., Chikaleke, V.A. and Thottappilly, G. (1992) Origin and Phylogeny of Guinea Yams as Revealed by RFLP Analysis of Chloroplast DNA and Nuclear Ribosomal DNA. Theoretical and Applied Genetics, 83, 743-751. https://doi.org/10.1007/BF00226693

[32] Mignouna, H.D., Abang, M.M. and Fagbemi, S.A. (2003) A Comparative Assessment of Molecular Marker Assays (AFLP, RAPD and SSR) for White Yam (Dioscorea rotundata) Germplasm Characterization. Annals of Applied Biology, 142, 269-276. https://doi.org/10.1111/j.1744-7348.2003.tb00250.x 
[33] Saski, C.A., Bhattacharjee, R., Scheffler, B.E. and Asiedu, R. (2015) Genomic Resources for Water Yam (Dioscorea alata L.): Analyses of EST Sequences, de Novo Sequencing and GBS Libraries. PLOS ONE, 10, e0134031. https://doi.org/10.1371/journal.pone.0134031

[34] Akakpo, R., Scarcelli, N., Chaïr, H., Dansi, A., Djedatin, G., Thuillet, A.-C., Rhoné, B., François, O., Alix, K. and Vigouroux, Y. (2017) Molecular Basis of African Yam Domestication: Analyses of Selection Point to Root Development, Starch Biosynthesis, and Photosynthesis Related Genes. BMC Genome, 18, 782.

https://doi.org/10.1186/s12864-017-4143-2

[35] Kress, W.J. and Erickson, D.L. (2007) A Two-Locus Global DNA Barcode for Land Plants: The Coding rbcL Gene Complements the Non-Coding trnH-psbA Spacer Region. PLOS ONE, 2, e508. https://doi.org/10.1371/journal.pone.0000508

[36] Schaefer, J. and Strimmer, K. (2005) An Empirical Bayes Approach to Inferring Large-Scale Gene Association Networks. Bioinformatics, 21, 754-764. https://doi.org/10.1093/bioinformatics/bti062

[37] Miller, S.E. (2007) DNA Barcoding and the Renaissance of Taxonomy. Proceeding of National Academy of Science, 104, 4775-4776.

https://doi.org/10.1073/pnas.0700466104

[38] Asahina, H., Shinozaki, J., Masuda, K., Morimitsu, Y. and Satake, M. (2010) Identification of Medicinal Dendrobium Species by Phylogenetic Analyses Using Matk and rbcL Sequences. Journal of the National Medical Association, 64, 133-138. https://doi.org/10.1007/s11418-009-0379-8

[39] Gao, X., Zhu, Y.-P., Wu, B.-C., Zhao, Y.-M., Chen, J.-Q. and Hang, Y.Y. (2008) Phylogeny of Dioscorea Sect. Stenophora Based on Chloroplast matK, rbcL and trnL-F Sequences. Journal of Systematic Evolution, 46, 315-321.

[40] DNA Learning Center Barcoding 101 (2011). http://www.dnabarcoding101.org/lab/protocol-2.html

[41] Bousalem, M., Durand, O., Scarcelli, N., Lebas, B.S.M., Kenyon, L., Marchandm, J.L., Lefort, F. and Seal, S.E. (2009) Dilemmas Caused by Endogenous Pararetroviruses Regarding the Taxonomy and Diagnosis of Yam (Dioscorea spp.) Badnaviruses: Analyses to Support Safe Germplasm Movement. Archives of Virology, 154, 297-314. https://doi.org/10.1007/s00705-009-0311-2

[42] Chen, S., Yao, H., Han, J., Liu, C., Song, J., Shi, L., Zhu, Y., Ma, X., Gao, T., Pang, X., Luo, K., Li, Y., Li, X., Jia, X., Lin, Y. and Leon, C. (2010) Validation of the ITS2 Region as a Novel DNA Barcode for Identifying Medicinal Plant Species. PLoS $O N E$, 5, e8613. https://doi.org/10.1371/journal.pone.0008613

[43] Tamura, K., Peterson, D., Peterson, N., Stecher, G., Nei, M. and Kumar, S. (2013) MEGA6: Molecular Evolutionary Genetics Analysis Using Maximum Likelihood, Evolutionary Distance, and Maximum Parsimony Methods. Molecular Biology Evolution, 28, 2731-2739. https://doi.org/10.1093/molbev/msr121

[44] Steel, M. and Penny, D. (2000) Parsimony, Likelihood, and the Role of Models in Molecular Phylogenetics. Molecular Biology Evolution, 17, 839-850.

https://doi.org/10.1093/oxfordjournals.molbev.a026364

[45] Kuck, P., Mayer, C., Wagele, J.W. and Misof, B. (2012) Long Branch Effects Distort Maximum Likelihood Phylogenies in Simulations Despite Selection of the Correct Model. PLoS ONE, 7, e36593. https://doi.org/10.1371/journal.pone.0036593

[46] Felsenstein, J. (1985) Evolutionary Trees from DNA Sequences: A Maximum Likelihood Approach. Molecular Biology Evolution, 17, 368-376.

https://doi.org/10.1007/BF01734359 
[47] Soininen, E.M., Valentini, A., Coissac, E., Miquel, C., Gielly, L., Brochmann, C., Brysting, A.K., Sønstebø, J.H., Ims, R.A., Yoccoz, N.G. and Taberlet, P. (2009) Analysing Diet of Small Herbivores: The Efficiency of DNA Barcoding Coupled with High-Throughput Pyrosequencing for Deciphering the Composition of Complex Plant Mixtures. Frontier Zoology, 6, 16. https://doi.org/10.1186/1742-9994-6-16

[48] Fazekas, A.J., Burgess, K.S., Kesanakurti, P.R., Graham, S.W., Newmaster, S.G., Husband, B.C., Percy, D.M., Hajibabaei, M. and Barrett, S.C. (2008) Multiple Multilocus DNA Barcodes from the Plastid Genome Discriminate Plant Species Equally Well. PLoS ONE, 3, e2802. https://doi.org/10.1371/journal.pone.0002802

[49] Hollingsworth, P.M., Graham, S.W. and Little, D.P. (2011) Choosing and Using a Plant DNA Barcode. PLoS ONE, 6, e19254. https://doi.org/10.1371/journal.pone.0019254

[50] Clement, W.L. and Donoghue, M.J. (2012) Barcoding Success as a Function of Phylogenetic Relatedness in Viburnum, a Clade of Woody Angiosperms. BMC Evolutionary Biology, 12, 73. https://doi.org/10.1186/1471-2148-12-73

[51] Li, X.W., Yang, Y., Henry, R.J., Rosetto, M., Wang, Y.T. and Chen, S.L. (2014) Plant DNA Barcoding, from Gene to Genome. Biological Reviews, 90, 157-166. https://doi.org/10.1111/brv.12104

[52] Dong, W., Xu, C., Li, C., Sun, J., Zuo, Y., Shi, S., Cheng, T., Guo, J. and Zhou, S. (2015) ycf1, the Most Promising Plastid DNA Barcode of Land Plants. Scientific Reports, 5, Article No. 8348. https://doi.org/10.1038/srep08348

[53] CBOL Plant Working Group (2009) A DNA Barcode for Land Plants. Proceedings of the National Academy of Sciences, 106, 12794-12797. https://doi.org/10.1073/pnas.0905845106

[54] Sun, X.Q., Zhu, Y.J., Guo, J.L., Peng, B., Bai, M.M. and Hang, Y.Y. (2012) DNA Barcoding the Dioscorea in China, a Vital Group in the Evolution of Monocotyledon: Use of matK Gene for Species Discrimination. PLoS ONE, 7, e32057. https://doi.org/10.1371/journal.pone.0032057

[55] Faith, D.P. and Baker, A.M. (2006) Phylogenetic Diversity (PD) and Biology Conservation: Some Bioinformatics Challenges. Evolutionary Bioinformatics, 2, 70-77. https://doi.org/10.1177/117693430600200007

[56] Mooers, A.O., Heard, S.B. and Chrostowski, E. (2005) In Phylogeny and Conservation. Oxford University Press, Oxford, 333.

[57] Faith, D.P. (2016) The Phylogenetic Diversity Framework: Linking Evolutionary History to Feature Diversity for Biodiversity Conservation. Biodiversity Conservation and Phylogenetic Systematics, Topics in Biodiversity and Conservation, 14. https://doi.org/10.1007/978-3-319-22461-9_3

[58] Cadotte, M.W. (2013) Experimental Evidence That Evolutionarily Diverse Assemblages Result in Higher Productivity. PNAS, 110, 8996-9000.

https://doi.org/10.1073/pnas.1301685110

[59] Pearson, W.R. (2014) BLAST and FASTA Similarity Searching for Multiple Sequence Alignment. Methods Molecular Biology, 1079, 75-101. https://doi.org/10.1007/978-1-62703-646-7_5

[60] Maloukh, L., Kumarappan, A., Jarrar, M., Salehi, J., El-waki, H. and Lakshmi, T.V.R. (2017) Discriminatory Power of $r b c L$ Barcode Locus for Authentication of Some of United Arab Emirates (UAE) Native Plants. 3 Biotech, 7, 144. https://doi.org/10.1007/s13205-017-0746-1

[61] Elansary, O.H., Ashfaq, M., Ali, H.M. and Yessoufou, K. (2017) The First Initiative 
of DNA Barcoding of Ornamental Plants from Egypt and Potential Applications in Horticulture Industry. PLoS ONE, 12, e0172170.

https://doi.org/10.1371/journal.pone.0172170

[62] Sikic, K. and Carugo, O. (2010) Protein Sequence Redundancy Reduction: Comparison of Various Methods. Bioinformatics, 5, 234-239.

https://doi.org/10.6026/97320630005234 


\section{Supplementary File 1}

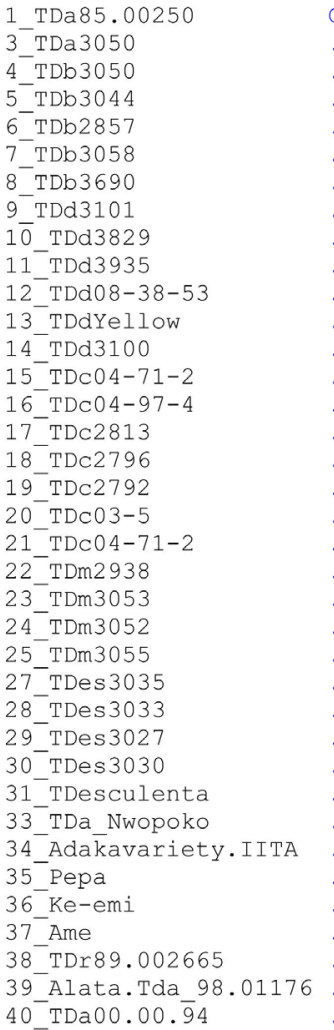

41 Tda00.00600

42 Ogojavariety. 1

43_GbanguVariety.1

44_obioturuguVariety.

45_Amolavariety. 1

46 Oginivariety

47-Damieha

48-Aloshivariety. 1

$49^{-}$IghuUna

51 Alata.2

52_Ighu_Dumenturum

53-Ighu

54 IghuUna.2

57--2-WhiteYam-_Iyo

59-D10WhiteNwopokoAdak

$60^{-}$D1Water-Nbanal

61 B-6-EDO

62_3threeLeavedYamono

65-WaterYam. Nbana

68 9ENEGBE

71_D1WaterYam-Nbana2

72_WaterYam-_Nbana

73_Wateryamji_mbala

$76^{-}$ona TDd

78_obel̄a

80_UtekpeVariety_2

81 WhiteYam-Nwoopoko

82_Yellowyam_Akpukpu

83-WaterYam-_Mbuna

84_BitterYam-Iwu_obe

85 AerialYam Edugbe

86_3Leaved_Yàm_ona

87_WaterYam-Mbana

89 WhiteYam Nwoopoko

$90^{-}$Yellowyam Oku

91_TrifoliateYam_TDd

92-ChineseYam TDes

93_YellowYam_TDes
90

100

110

120

130

140

150

160

CCGCCGAATCGTCCACCGGTACATGGACAACTGTGTGGACTGATGGACTTACCAGTCTTGATCGTTACAAAGGACGATGC

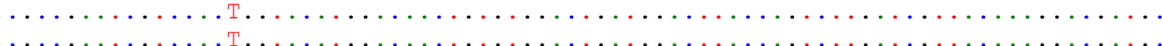

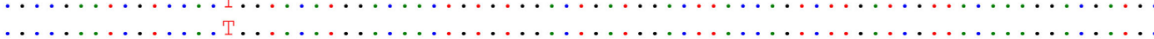

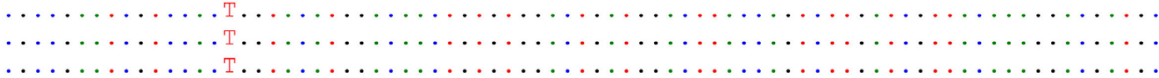

.

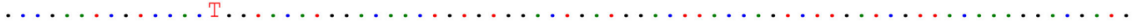

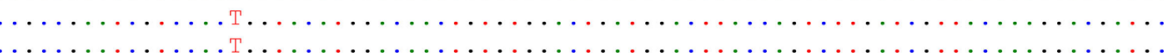

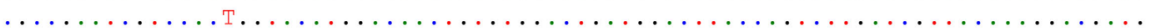

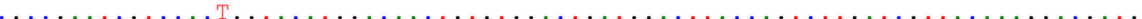

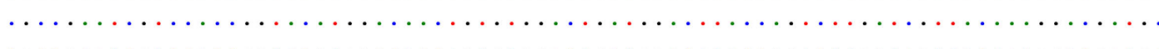

.

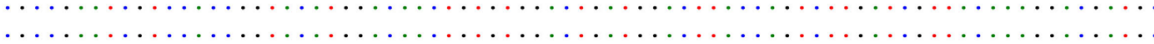

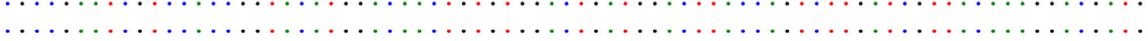

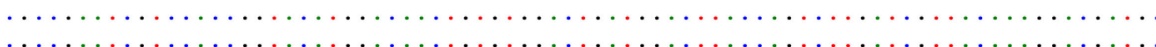

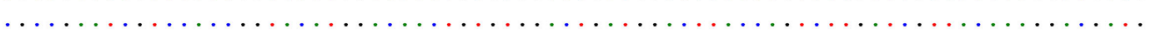

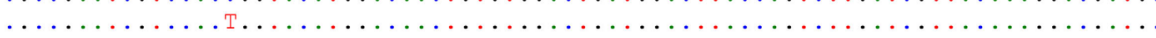

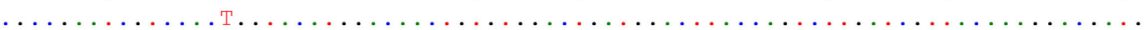

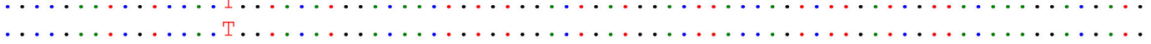

- .

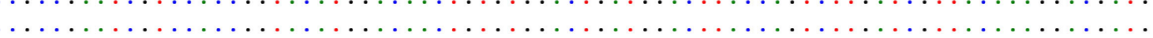



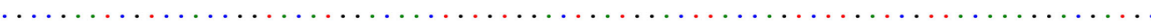

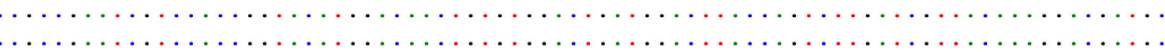

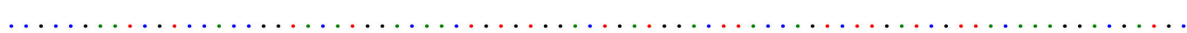

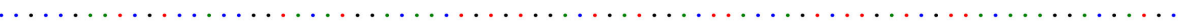

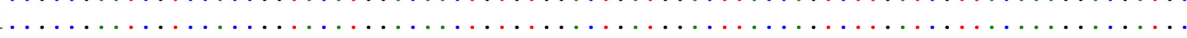

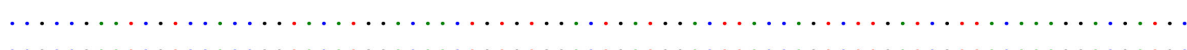

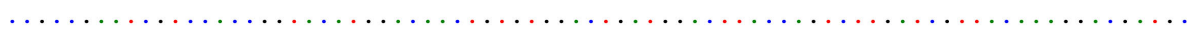

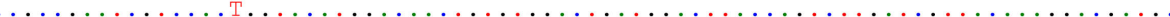

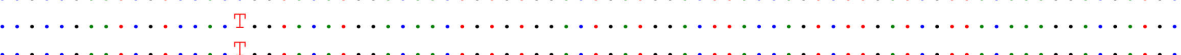

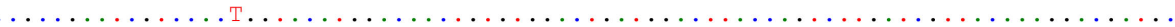

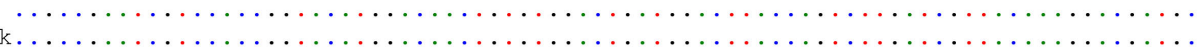

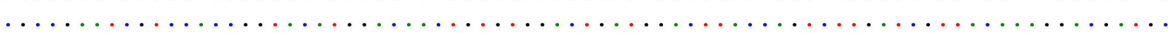

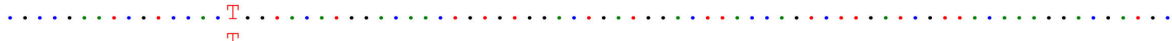

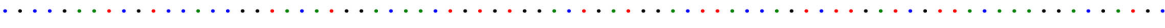

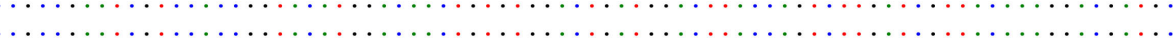

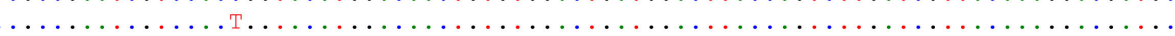

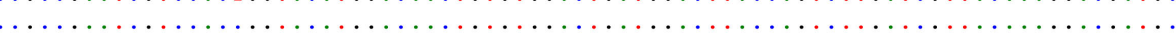

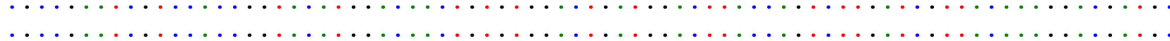

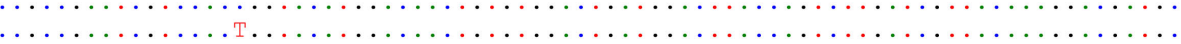

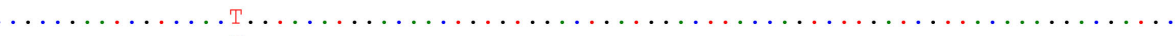

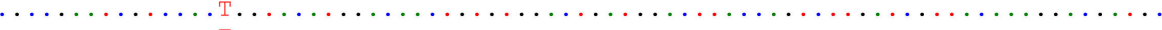

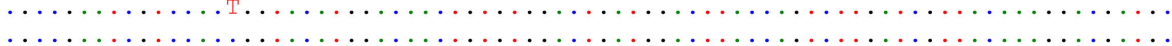

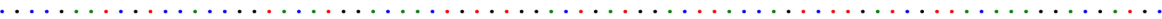

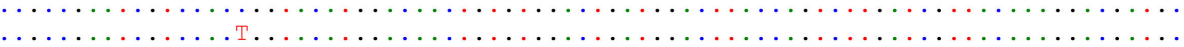

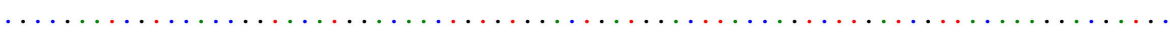




\section{Supplementary File 2}

Table S1. List of sequenced yam species collected from different locations and their GenBank accession numbers.

\begin{tabular}{|c|c|c|c|c|}
\hline Sample IDs & Location & LGA & State & GenBank No \\
\hline 1_TDa85.00250 & IITA & Akinyele & Oyo & MH078115 \\
\hline 3_TDa3050 & IITA & Akinyele & Oyo & MH078154 \\
\hline 4_TDb3050 & IITA & Akinyele & Oyo & MH078155 \\
\hline 5_TDb3044 & IITA & Akinyele & Oyo & MH078156 \\
\hline 6_TDb2857 & IITA & Akinyele & Oyo & MH078157 \\
\hline 7_TDb3058 & IITA & Akinyele & Oyo & MH078158 \\
\hline 8_TDb3690 & IITA & Akinyele & Oyo & MH078159 \\
\hline 9_TDd3101 & IITA & Akinyele & Oyo & MH078163 \\
\hline 10_TDd3829 & IITA & Akinyele & Oyo & MH078164 \\
\hline 11_TDd3935 & IITA & Akinyele & Oyo & MH078165 \\
\hline 12_TDd08-38-53 & IITA & Akinyele & Oyo & MH078166 \\
\hline 13_TDdYellow & IITA & Akinyele & Oyo & MH078167 \\
\hline 14_TDd3100 & IITA & Akinyele & Oyo & MH078168 \\
\hline 15_TDc0471-2 & IITA & Akinyele & Oyo & MH078170 \\
\hline 16_TDc0497-4 & IITA & Akinyele & Oyo & MH078127 \\
\hline 17_TDc2813 & IITA & Akinyele & Oyо & MH078141 \\
\hline 18_TDc2796 & IITA & Akinyele & Oyo & MH078142 \\
\hline 19_TDc2792 & IITA & Akinyele & Oyo & MH078171 \\
\hline 20_TDc03-5 & IITA & Akinyele & Oyo & MH078143 \\
\hline 21_TDc04-71-2 & IITA & Akinyele & Oyo & MH078144 \\
\hline 22_TDm2938 & IITA & Akinyele & Oyo & MH078128 \\
\hline 23_TDm3053 & IITA & Akinyele & Oyo & MH078145 \\
\hline 24_TDm3052 & IITA & Akinyele & Oyo & MH078146 \\
\hline 25_TDm3055 & IITA & Akinyele & Oyo & MH078172 \\
\hline 27_TDes3035 & IITA & Akinyele & Oyo & MH078175 \\
\hline 28_TDes3033 & IITA & Akinyele & Oyo & MH078176 \\
\hline 29_TDes 3027 & IITA & Akinyele & Oyo & MH078177 \\
\hline 30_TDes 3030 & IITA & Akinyele & Oyo & MH078178 \\
\hline 31_TDesculenta & IITA & Akinyele & Oyo & MH078179 \\
\hline 33_TDaNwokporo & IITA & Akinyele & Oyo & MH078116 \\
\hline 34_Adakavariety & IITA & Akinyele & Oyo & MH078117 \\
\hline 35_Pepa & IITA & Akinyele & Oyo & MH078129 \\
\hline 36_Ke-emi & IITA & Akinyele & Oyo & MH078130 \\
\hline 37_Ame & IITA & Akinyele & Oyo & MH078131 \\
\hline 38_TDr 89.002665 & IITA & Akinyele & Oyo & MH078132 \\
\hline 39_AlataTda 98.01176 & IITA & Akinyele & Oyo & MH078133 \\
\hline 40_TDa00.00.94 41_Alata & IITA & Akinyele & Oyo & MH078134 \\
\hline
\end{tabular}




\section{Continued}

\begin{tabular}{|c|c|c|c|c|}
\hline 41_Tda00.00600 & IITA & Akinyele & Oyо & MH078147 \\
\hline 42_OgojaVariety.1 & IITA & Akinyele & Oyo & MH078135 \\
\hline 43_Gbangu_Variety.1 & IITA & Akinyele & Oyo & MH078188 \\
\hline 44_ObioturuguVariety.1 & IITA & Akinyele & Oyo & MH078136 \\
\hline 45_AmolaVariety .1 & IITA & Akinyele & Oyо & MH078137 \\
\hline 46_OginiVariety & IITA & Akinyele & Oyo & MH078173 \\
\hline 47_Damieha & IITA & Akinyele & Oyo & MH078138 \\
\hline 48_Aloshivariety.1 & IITA & Akinyele & Oyo & MH078139 \\
\hline 49_IghuUna & Osonu & Ezeagu & Enugu & MH078184 \\
\hline 51_Alata2 & Osonu & Ezeagu & Enugu & MH078118 \\
\hline 52_Ighu_Dumenturum & Osonu & Ezeagu & Enugu & MH078185 \\
\hline 53_Ighu & Osonu & Ezeagu & Enugu & MH078182 \\
\hline 54_IghuUna.2 & Osonu & Ezeagu & Enugu & MH078183 \\
\hline 57_2-WhiteYam- Iyo & Ukaka Ngwo & Enugu North & Enugu & MH078148 \\
\hline 59_D10WhiteNwopoko-Adaka & Agbalenyi Nachi & Oji-River & Enugu & MH078114 \\
\hline 60_D1Water-Nbana1 & Agbalenyi Nachi & Oji-River & Enugu & MH078119 \\
\hline 61-6- EDO & Ukaka Ngwo & Enugu North & Enugu & MH078160 \\
\hline 62_3LeavedYam-Ono & Ukaka Ngwo & Enugu North & Enugu & MH078180 \\
\hline 65_WaterYam.Nbana & Ukaka Ngwo & Enugu North & Enugu & MH078120 \\
\hline 68_9ENEGBE & Ndibinagu Umuaga & Udi & Enugu & MH078149 \\
\hline 71_D1WaterYam-Nbana2 & Agbalenyi Nachi & Oji-River & Enugu & MH078121 \\
\hline 72_1-Water_Yam-_Nbana & Ndibinagu Umuaga & Udi & Enugu & MH078122 \\
\hline 73_Wateryamji_mbala & Nkalagu & Ishielu & Ebonyi & MH078123 \\
\hline 76_OnaTDd & Ezzamgbo & Ohaukwu & Ebonyi & MH078181 \\
\hline 78_Obella & Ezzamgbo & Ohaukwu & Ebonyi & MH078140 \\
\hline 80_UtekpeVariety_2 & Ezzamgbo & Ohaukwu & Ebonyi & MH078174 \\
\hline 81_WhiteYam-Nw-opoko & Amaeke Amaigbo Ozalla & Nkanu West & Enugu & MH078150 \\
\hline 82_Yellowyam_Akpukpu & Amaeke Amaigbo Ozalla & Nkanu West & Enugu & MH078151 \\
\hline 83_WaterYam- Mbuna & Amaeke Amaigbo Ozalla & Nkanu West & Enugu & MH078161 \\
\hline 84_BitterYam-Iwu_obe & Amaeke Amaigbo Ozalla & Nkanu West & Enugu & MH078169 \\
\hline 85_AerialYam_Edugbe & Amaeke Amaigbo Ozalla & Nkanu West & Enugu & MH078162 \\
\hline 86_3LeavedYam_Ona & Ede Oballa & Nsukka & Enugu & MH078186 \\
\hline 87_WaterYam-Mbana & $\mathrm{Nru}$ & Nsukka & Enugu & MH078124 \\
\hline 89_WhiteYam_Nwopoko & Ibagwa Aka & Igboeze South & Enugu & MH078152 \\
\hline 90_Yellowyam_Oku & Ihe Owerre & Nsukka & Enugu & MH078125 \\
\hline 91_TrifoliateYam_TDb & Ukana & Udi & Enugu & MH078187 \\
\hline 92_ChineseYam_TDes & Ukana & Udi & Enugu & MH078126 \\
\hline 93_YellowYam_TDes & Ukana & Udi & Enugu & MH078153 \\
\hline
\end{tabular}

IITA = International Institute of Tropical Agriculture; LGA = Local Government Authority. 